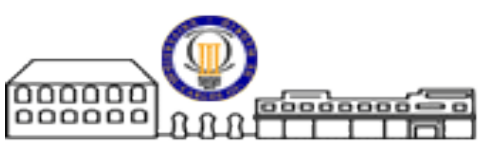

Working Paper

Economic Series 16-03

November 2016

ISSN 2340-5031
UNIVERSIDAD CARLOS III DE MADRID

Departamento de Economía

Universidad Carlos III de Madrid

C/ Madrid, 126, 28903 Getafe (Spain)

Fax (34) 916249875

\title{
“THE IMPACT OF CLASSIFICATION SYSTEMS IN THE EVALUATION OF THE RESEARCH PERFORMANCE OF THE LEIDEN RANKING UNIVERSITIES"
}

\author{
Antonio Perianes-Rodriguez ${ }^{a}$ and Javier Ruiz-Castillo ${ }^{b}$ \\ ${ }^{a}$ Departamento de Biblioteconomía y Documentación, Universidad Carlos III, \\ SCImago Research Group \\ bDepartamento de Economía, Universidad Carlos III
}

\begin{abstract}
In this paper, we investigate the consequences of choosing different classification systems namely, the way publications (or journals) are assigned to scientific fields- for the ranking of research units. We study the impact of this choice on the ranking of 500 universities in the 2013 edition of the Leiden Ranking in two cases. Firstly, we compare a Web of Science journal-level classification system, consisting of 236 subject categories, and a publication-level algorithmically constructed system, denoted G8, consisting of 5,119 clusters. The result is that the consequences of the move from the WoS to the G8 system using the Top 1\% citation impact indicator are much greater than the consequences of this move using the Top 10\% indicator. In the second place, we compare the G8 classification system and a publication-level alternative of the same family, the G6 system, consisting of 1,363 clusters. The result is that, although less important than in the previous case, the consequences of the move from the G6 to the G8 system under the Top 1\% indicator are still of a large order of magnitude.
\end{abstract}

Acknowledgements. This is the second version of the Working Paper with the same title published in this series in January 2016. This research project builds on earlier work started by Antonio PerianesRodriguez during a research visit to the Centre for Science and Technology Studies (CWTS) of Leiden University as awardee of José Castillejo grant, CAS15/00178, funded by the Spanish MEC. RuizCastillo is a visiting researcher at CW'TS and gratefully acknowledges CWTS for the use of its data. Ruiz-Castillo acknowledges financial support from the Spanish MEC through grant ECO2014-55953P, as well as grant MDM 2014-0431 to his Departamento de Economía. 


\section{INTRODUCTION}

Assume we want to compare the performance of a set of research units. This is, of course, a complex task that depends on a large number of factors. For example, in a recent review article, Waltman (2016) discusses the choice of a bibliometric database, the selection of publications and citations (document type, language, national versus international journals, self-citations, citation window), counting methods for dealing with co-authored publications and, last but not least, citation impact indicators. Assume that these problems have been solved, that is, assume that we have information, collected from a reliable bibliometric database, on the citation distributions of the articles in the periodical literature that these research units have published during a certain period of time in a number of scientific fields. Assume also that we have satisfactorily solved the assignment of coauthored publications to the set of research units in question, and that we have selected some appropriate citation impact indicator. In this paper, we study the consequences of choosing between two classification systems of science, that is, between two ways of assigning individual publications (or journals) to scientific fields.

This is an important problem, since differences in production and citation practices between scientific fields must be normalized before any meaningful assessment of these units' research output is possible. As also explained in Waltman (2016), barring source (citing-side) normalization procedures (see inter alia Waltman et al., 2013, and the references cited there), any other field normalization procedure will depend on the classification system used.

We consider two types of classification systems: the Web of Science (WoS hereafter) journallevel classification system, consisting of 236 journal subject categories (or simply categories hereafter), and an alternative publication-level system arising from the algorithmic methodology introduced in Waltman \& Van Eck (2012) that classifies individual publications into clusters solely based on direct citations among them. 
In practice, the choice of the WoS classification system is often made because, together with the Scopus system, it is readily available. However, a number of studies question the appropriateness of this system for normalization purposes. ${ }^{1}$ Among the publication-level alternatives, Klavans and Boyack (2015) conclude that classification systems based on direct citation using the Waltman \& Van Eck (2012) methodology are more accurate than classification systems based on bibliographic coupling or co-citation. Ruiz-Castillo \& Waltman (2015) apply the Waltman \& Van Eck (2012) methodology to a WoS dataset consisting of 9.4 million publications from the 2003-2012 period. A sequence of twelve independent classification systems is obtained, in each of which the same set of publications is assigned to an increasing number of clusters. In this paper, we select the two members of this sequence corresponding to granularity levels 6 and 8 (the G6 and G8 classification systems hereafter) consisting of 1,363 and 5,119 clusters.

In this scenario, we focus on the comparison between the WoS and the G8 systems that were already studied in Ruiz-Castillo \& Waltman (2015). However, to emphasize the potential importance of the granularity level choice, we also compare the G6 and G8 publication-level systems that have been also studied in Perianes-Rodriguez \& Ruiz-Castillo (2016a).

As indicated before, among other factors, ranking research units critically depends on the choice of a citation impact indicator. In this paper, we focus on a percentile rank indicator for two reasons. Firstly, although percentile rank indicators directly incorporate a suitable normalization procedure for citation counts of publications from different clusters or categories (see inter alia Bornmann \& Marx, 2013), it is important to understand that they are conditional on the classification system used. For example, given any classification system with $J$ clusters, the Top $X \%$ indicator is defined as the percentage of an institution's scientific output included in the set formed by the top $X \%$ of the most

\footnotetext{
1 See inter alia Neuhaus \& Daniel (2009) for Chemistry and related fields, Van Leeuwen \& Calero Medina (2012) for Economics \& Business, Van Eck et al. (2013) for Clinical and Basic Medical Research, and Leydesdorff \& Bornman (2015) for Library and Information Science, and Science \& Technology Studies.
} 
cited publications in each of the $J$ clusters. The ranking of units obtained with this classification system will be different from the ranking obtained with the same TopX\% indicator but a different classification system consisting of $K$ clusters with $K \neq J$.

Secondly, percentile rank indicators occupy a prominent role both in the Leiden Ranking of universities and the SCImago Institutions Ranking. ${ }^{2}$ Together with the Mean Normalized Citation Score, the 2015 and 2016 versions of the Leiden Ranking include the Top 50\%, the Top 10\%, and the Top 1\% indicators, whereas the SCImago Institutions Ranking includes the Top 10\% indicator. Since Ruiz-Castillo \& Waltman (2015) studied the consequences of using the Top 10\% indicator under the WoS and the G8 systems, in this paper we study the consequences of using the Top 1\% indicator. This is an interesting contrast, since it is well known that differences between classification systems tend to increase as the threshold of excellence goes up (Zitt et al., 2005, and Perianes-Rodriguez \& RuizCastillo, 2016a).

To facilitate the comparison of the consequences of using the Top $10 \%$ or the Top $1 \%$ indicators, in this paper we follow closely the following four methodological decisions adopted in Ruiz-Castillo \& Waltman (2015). (i) We focus on the 3.6 million articles published in the 2005-2008 period, and the citations they receive during a five-year citation window for each year in that period. (ii) In the WoS system, approximately $45 \%$ of the articles in the dataset are assigned to two or more categories up to a maximum of six. To deal with this problem, we adopt a fractional strategy. (iii) The set of research units investigated consists of the 500 universities included in the 2013 edition of the CWTS Leiden Ranking (Waltman et al., 2012a) -the LR universities hereafter. (iv) There are 2.4 million distinct articles in which at least one author belongs to one of the LR universities. Only $30 \%$ of this total has a

\footnotetext{
2 The Leiden Ranking (www.leidenranking.com) is a university ranking compiled by the Centre for Science and Technology Studies (CWTS), Leiden University, The Netherlands, using the Thomson Reuters bibliographic database Web of Science as the source of the publication and citation data. SCImago is a research group from the Consejo Superior de Investigaciones Cientificas, University of Granada, Extremadura, Carlos III (Madrid) and Alcalá de Henares in Spain. The SCImago Institutions Rankings (SIR; www.scimagoir.com) is a bibliometric ranking of research institutions based on Elsevier's Scopus database.
} 
single address line. We use the address-line fractional counting approach to solve the problem of the remaining publications assigned to several co-authors working in different institutions. As a result, the total number of articles in the LR universities becomes 1.9 million. (v) To solve the problems in the computation of the indicators generated by the discrete nature of citation distributions combined with the presence of many publications with the same number of citations, we follow the approach recommended in Waltman \& Schreiber (2013).

The rest of the paper is organized into three Sections and an Appendix. Section II serves two purposes: it presents the data, as well as the results concerning the differences between the three classification systems as the threshold of excellence increases. Section III contains the empirical results concerning the consequences of applying the Top 1\% indicator to the 500 LR universities under the three classification systems. Finally, Section IV offers some conclusions. The Appendix includes two items: the method for establishing the differences between the WoS and the G8 systems when the WoS system is constructed according to a fractional scheme, and the ranking of the LR universities according to the Top $1 \%$ indicator under the three classification systems.

\section{DATA, AND DIFFERENCES BETWEEN CLASSIFICATION SYSTEMS}

\section{II.1. The data}

Our dataset results from the application of the publication-level algorithmic methodology in Waltman \& Van Eck (2012) to 9,446,622 distinct articles published in 2003-2012. Publications in local journals, as well as popular magazines and trade journals, have been excluded (for the details, see RuizCastillo \& Waltman, 2015). We work with journals in the sciences, the social sciences, and the arts and humanities, although many arts and humanities journals are excluded because they are of a local nature.

In this paper, we focus on the set of 3,614,447 distinct articles published in the period 20052008. To save space, descriptive statistics for this dataset are available in Ruiz-Castillo \& Waltman 
(2015). To deal with the problem of multiple assignment of articles to WoS categories, we adopt a fractional strategy according to which each article assigned to, say, $m$ categories, is fractioned into $m$ equal pieces, with each piece assigned to each of the $m$ categories.

\section{II.2. Differences between classification systems}

Following Zitt et al. (2005), we consider the possibility of computing the set of the top $X \%$ most cited publications in every cluster in a pair of classification systems A and B. An article that belongs to the top $X^{\%} \%$ in cluster $j$ in system $\mathrm{A}$ (or $\mathrm{B}$ ) may not belong to the top $X^{\%} \%$ in cluster $l$ in system $\mathrm{B}$ (or A). The more often this is the case, the more different the two systems will be according to the $X^{\%}$ criterion.

We begin by studying the differences between the G8 and the G6 systems. Let $c_{j}$ be the ordered citation distribution of cluster $j$ in system $\mathrm{G} 8$, where $j=1, \ldots, 5119$. The union $C=\cup_{j}\left\{c_{j}\right\}$ is the overall citation distribution in the all-sciences case. Similarly, let $d_{g}$ be the ordered citation distribution of cluster $g$ in system G6, where $g=1, \ldots, 1363$, and let $D=\cup_{g}\left\{d_{g}\right\}$ be the overall citation distribution in this case. The total number of articles in $C$, and $D$ is 3.6 million. For the comparison between the G6 and G8 systems, let $x_{j}$ and $x_{g}$ be the sets of the top X\% most cited articles in cluster citation distributions $c_{j}$ and $c_{g}$, and denote the union of these sets by $X^{G 8}=\cup_{j}\left\{x_{j}\right\}$, and $X^{G 6}=\cup_{g}$ $\left\{x_{g}\right\}$. Since both systems have the same total number of articles, the number of articles in $X^{G 8}$ and $X^{G 6}$ is also the same, say $N^{X}$. Let $X^{68}$ be the set of distinct articles common to both systems, namely, let $X^{68}=X^{G 6} \cap X^{G 8}$. Let $N^{X 68}$ be the number of articles in $X^{68}$. The difference between the top $X \%$ most cited articles in both systems is measured through the percentage that the articles in $X^{G 8}-$

$X^{68}$ represent in $X^{G 8}$ (or the percentage that the articles in $X^{G 6}-X^{68}$ represent in $X^{G 6}$ ), that is, 
through the expression $100\left(N^{X}-N^{X 68}\right) / N^{X}$. The results for the top $50 \%, 10 \%$, and $1 \%$ most cited articles in the dataset with 3.6 million articles are in Table 1.

\section{Table 1 around here}

Let $e_{k}$ be the ordered citation distribution of category $k$ in the WoS system, where $k=1, \ldots$, 236. The union $E=\cup_{k}\left\{e_{k}\right\}$ is the overall citation distribution in this case. Although the number of articles in each citation distribution $e_{k}$ will typically be a fractional number, the total number of articles in $E$ is 3.6 million. However, to compare the systems G8 and G6 with the WoS system we must take into account that the latter has been constructed according to a fractional scheme. To facilitate the reading of the text, the extension of the original method can be found in the Appendix. The results for the comparison between the WoS and the two granularity levels are also in Table 1.

Table 1 warrants the following two comments. Firstly, independently of the pair of classification systems being compared, we confirm that -as in Zitt et al. (2005) and Perianes-Rodriguez \& RuizCastillo (2016a) - the difference between them increases as the threshold of excellence goes up. Secondly, in the key comparison between the WoS and the G8 systems we observe that, at least in the upper tail of the cluster and categories citation distributions, the difference is very large. In particular, for the top $1 \%$ of most cited articles this difference is $51.7 \%$, or twenty percentage points above the difference for the top $10 \%$ of most cited articles. When comparing the G6 and G8 systems, this difference is smaller $-31.1 \%$ - but non-negligible at all. ${ }^{3}$ Therefore, the choice between classification systems could have dramatic consequences for the ranking of research units when the citation impact indicator used in practice is defined over the very upper tail of citation distributions. This is certainly the case for the Top 1\% indicator used in this paper (Section III below).

\footnotetext{
${ }^{3}$ Using the same dataset, Perianes-Rodriguez \& Ruiz-Castillo (2016a) make the same comparisons when the problem of the assignment of articles to multiple categories in the WoS system is solved according to a multiplicative strategy. Differences between the WoS and the publication-level systems for every $X=50 \%, 10 \%$, and $1 \%$ are of the same order of magnitude as those shown in Table 1.
} 
There are 2,420,054 distinct articles -or 67\% of the 3.6 million articles published in 2005-2008with at least one address line belonging to a LR university. Differences between the three classification systems for this dataset according to the top $X \%$ criterion when $X=50 \%, 10 \%$, and $1 \%$, are essentially the same as the differences presented in Table 1(results are available on request).

\section{II.3. Counting method}

The distribution of the 2.4 million distinct articles with at least one address line belongs to an LR university by the number of address lines, as well as other descriptive statistics can be found in Perianes-Rodriguez \& Ruiz-Castillo (2016b). It turns out that the percentage of articles with a single address line is $30.0 \%$ of the total. The assignment of the remaining $70 \%$ of the 2.4 million distinct articles that are co-authored by two or more institutions is made according to the address-level fractional counting method. The total number of articles in the LR universities according to this method is $1,886,106.1$, or $77.9 \%$ of the 2.4 million articles with at least one address line belonging to a LR university, and 52.2\% of the 3.6 million articles published in 2005-2008 (see Perianes-Rodriguez \& Ruiz-Castillo, 2015a, for the distribution of the 1.9 million articles among the 500 LR universities).

\section{EMPIRICAL RESULTS}

\section{III.1. The WoS versus the G8 classification systems}

Columns 1 and 2 in Table A in the Appendix present the results of applying the Top 1\% indicator in the WoS and G8 classification systems, ordered by the Top 1\% values under the G8 system. The university ranks according to the Top 1\% values in the WoS system, the ranking differences, and the differences between the indicator values are presented in column 3, 4, and 5, respectively. ${ }^{4}$ We begin by observing that the Pearson and the Spearman correlation coefficients between university values and ranks according to the Top $1 \%$ indicator -0.92 and 0.90 - are

\footnotetext{
4 Table A reports the ratio of each university's Top 1\% value and the world reference, namely, $1.00 \%$. Thus, if a university has a Top $1 \%$ value of $1.12 \%$, Table A reports a value of $(1.12 \% / 1.00 \%)=1.12$, so that the value one can serve as a benchmark for evaluating the research units in the usual way.
} 
significantly lower than according to the Top 10\% indicator in Ruiz-Castillo \& Waltman (2015) -0.97

and 0.96, respectively. This anticipates greater differences between university rankings under the Top $1 \%$ indicator.

We take two aspects into account: the re-rankings that take place in the move from the WoS to the G8 system when the Top 1\% is used (column 4 in Table A), and the differences between the university values themselves (column 5 in Table A). The results, which are presented in Table 2, can be compared with the differences found in going from the WoS to the G8 system when using the Top 10\% indicator. To facilitate the comparison, the differences for the Top $10 \%$ indicator, taken from Table 6 in Ruiz-Castillo \& Waltman (2015), are presented in Table 3.

\section{Tables 2 and 3 around here}

Relatively large re-rankings of more than 25 positions according to the Top $1 \%$ indicator occur much more frequently than according to the Top 10\% indicator: 296 versus 195 universities are affected in each case (Panel $\mathrm{A}$ in Tables 2 and 3). This is mostly due to the situation among the last 400 universities, where these numbers are 264 and 188, respectively. Nevertheless, when using the Top $1 \%$ indicator, as many as 32 large re-rankings take place among the first 100 universities. On the other hand, only 61 universities, or $12.2 \%$ of the total exhibit small re-rankings equal to or smaller than five positions.

Something similar occurs when we consider differences in indicator values (Panel B in Tables 2 and 3). According to the Top 1\% indicator, large changes greater than 0.10 in indicator values occur with more or less equal frequency among the first 100 and the last 400 universities: in both cases, approximately $60 \%$ of universities exhibit large changes in indicator values. In contrast, these percentages are $13 \%$ and $20.5 \%$ according to the Top $10 \%$ indicator.

By way of example, Panel $\mathrm{C}$ in Table 2 includes the largest gainers and losers among the first 100 universities when going from the WoS to the G8 system. The three columns include the ranking 
according to the G8 system, the number of positions in the re-ranking, and the difference in Top 1\% values. With the criterion of a re-ranking greater than 50 positions, we have only 11 gainers. In nine cases the gains take place between positions 49 and 99. We have also added the loser with the greatest difference in Top 1\% values, namely, Rice University.

Finally, to illustrate the sensitivity of university Top 1\% values to the choice of a classification system, we use the 32 Chinese universities (excluding Hong Kong) as an interesting example. Each of these universities is indicated using a red cross in Figure 1. The main lesson is that, as we go from the WoS to the G8 system, the performance of almost all Chinese universities worsens. This deterioration is especially significant for the best performing Chinese universities. Not surprisingly, these results coincide with what we saw in Ruiz-Castillo \& Waltman (2015) when we use the Top 10\% indicator. An explanation of the Chinese case is beyond the scope of this paper, but it may relate to the specific characteristics of the research areas in which Chinese universities focus their activity or to the citation behavior of Chinese researchers (since researchers' citation behavior determines how publications are clustered in the algorithmic methodology).

\section{Figure 1 around here}

\section{III.2. The G6 versus the G8 classification systems}

This Sub-section investigates the consequences of using two different granularity levels within the sequence of publication-level options studied in Ruiz-Castillo \& Waltman (2015). Columns 1 and 2 in Table B in the Appendix present the results of applying the Top 1\% indicator in the G6 and G8 classification systems, ordered by the Top 1\% values under the G8 system. The university ranks according to the Top 1\% values in the G6 system, the ranking differences, and the differences between the indicator values are presented in column 3, 4, and 5, respectively. 
We begin by observing that the Pearson and the Spearman correlation coefficients between university values and ranks according to the Top 1\% indicator are 0.97 and 0.96 . The results on rerankings and differences between university values are presented in Table 4.

\section{Table 4 around here}

Three comments are in order. Firstly, relatively large re-rankings of more than 25 positions are less frequent than before: 222 versus 296 universities are affected in each case (Panel A in Tables 4 and 2). This reduction of approximately $15 \%$ cases is of the same order of magnitude among the first 100 and then last 400 universities. Nevertheless, only 30\% among the first 100 and $15.5 \%$ among the last 400 universities experience small re-rankings equal to or smaller than five positions. Thus, a large proportion of universities experience intermediate or large re-ranking in the move from the G6 and G8 systems. Secondly, large changes greater than 0.10 in indicator values are now approximately one half of what we observed before (Panel B in Tables 2 and 4). However, this is due to the situation among the last 400 universities, since 29 versus 31 of the first 100 universities are affected in the two moves. Thirdly, Panel $\mathrm{C}$ in Table 4 includes the largest gainers and losers among the first 100 universities when going from the G6 to the G8 system. As before, the three columns include the ranking according to the G8 system, the number of positions in the re-ranking, and the difference in Top 1\% values. There are only four rather than eleven universities among the gainers of more than 50 positions.

\section{DISCUSSION AND CONCLUSIONS}

There are a number of recent results favoring publication-level over journal-level classification systems. Using a set of new gold standards - consisting of articles with at least 100 references-,

Klavans \& Boyack (2015) compare publication-level algorithmically constructed classification systems based on direct citations, à la Waltman \& Van Eck (2012), with six journal-level systems that do not 
include the WoS. They conclude that the former are more accurate than the latter in the sense that they are better at concentrating references. Among the alternatives to the WoS system, Ruiz-Castillo \& Waltman (2015) study a sequence of twelve independent classification systems based on the Waltman \& Van Eck (2012) algorithmic methodology at different granularity levels. Although it is difficult to isolate an optimal granularity level, Ruiz-Castillo \& Waltman (2015) conclude that classification systems with a few thousands clusters with a minimum size provide a credible alternative to the WoS system, and focus their empirical analysis on the G8 system. Finally, consider the standard target (or cited-side) normalization procedure in which normalized citation scores in every cluster are equal to the original raw citations divided by the cluster mean citation. In Perianes-Rodriguez \& Ruiz-Castillo (2016a) we study the following criterion to compare a pair of classification systems A and B: if one could establish that the standard normalization procedure based on system A performs better than the standard normalization procedure based on system B, then we would recommend the use of system A over system B. According to this criterion, the G8 clearly dominates the G6 system. On the other hand, although the comparison of the G8- and WoS-based standard normalization procedures leads to mixed results (technically, the two classification systems turn out to be non-comparable), it can be said that the G8-normalization procedure performs better using the WoS system for evaluation purposes than the WoS-normalization procedure using the G8 system for evaluation purposes.

In practice, the CWTS adopts a publication-level algorithmically constructed classification system à la Waltman \& Van Eck (2012) consisting of 3,822 and 4,113 clusters in the 2015 and 2016 editions of the Leiden Ranking. Taking into account the above results, we sympathize with this choice. However, we believe that it is important to keep studying the consequences of moving from joint-level classification systems to publication-level alternatives. Among the latter, we also should bear in mind the consequences of choosing alternative granularity levels. 
This paper has contributed to this topic by comparing the consequences for the ranking of the 500 universities in the 2013 edition of the LR in two cases: using a WoS journal-level classification system with 236 categories versus the G8 publication-level system with 5,119 clusters, and using the G6 publication-level system with 1,363 clusters versus the G8 system of the same family. Naturally, the ranking of research units depends also on which citation impact indicator we use. Since Ruiz-Castillo \& Waltman (2015) studied the Top 10\% indicator, in this paper we have focused on the Top 1\% indicator.

The difference between the sets of top $10 \%$ most cited publications in every cluster/category in the G8 and WoS systems is considerable, namely, 31.7\%. However, this difference for the top 1\% most cited publications is twenty percentage points greater, namely, 51.7\%. Our first result follows from here: the consequences of the move from the WoS to the G8 system are much greater when using the Top 1\% than with the Top 10\% citation impact indicator. The difference between the sets of top 1\% most cited publications in every cluster in the G6 and G8 systems is considerable: $31.1 \%$. This helps explaining our second result: although less important than the consequences of the move from the WoS to the G8 system, the consequences of the move from the G6 to the G8 system are still of a large order of magnitude.

The conclusion is clear. Recent results indicate that there are good reasons to move from journal-level to publication-level algorithmically constructed classification systems. At the same time, due to the high skewness of citation distributions, we are witnessing a shift towards citation impact indicators defined over the very upper tail of citation distributions. Personally, we believe that both moves are advisable. However, the results of this paper indicate that this double trend has important consequences in two interesting cases. On one hand, the move from the WoS to the G8 system using the Top 1\% indicator changes the ranking of the 500 universities in the 2013 edition of the Leiden 
Ranking quite dramatically. On the other hand, when the citation impact indicator is the Top 1\%, this ranking -although to a lesser extent- is also considerably affected by the granularity level used. 


\section{REFERENCES}

Klavans, R., \& Boyack, K. W. (2015). Which type of citation analysis generates the most accurate taxonomy of scientific and technical knowledge? arXiv:1511.05078v1 [cs.DL].

Perianes-Rodriguez, A., and Ruiz-Castillo, J. (2016a). A comparison of two ways of evaluating research units working in different scientific fields. Scientometrics, 106, 539-561.

Perianes-Rodriguez, A., and Ruiz-Castillo, J. (2016b). University citation distributions. Journal of the American Society for Information Science and Technolog, 67, 2790-2804.

Ruiz-Castillo, J., and Waltman, L. (2015). Field-normalized citation impact indicators using algorithmically constructed classification systems of science. Journal of Informetrics, 9, 102-117.

Waltman, L. (2016). A review of the literature on citation impact indicators. Journal of Informetrics, 10, 365-391.

Waltman L., \& Van Eck, N. J. (2013). Source normalized indicators of citation impact: An overview of different approaches and an empirical comparison. Scientometrics, 96, 699-716.

Waltman, L., Calero-Medina, C., Kosten, J., Noyons, E. C. M., Tijssen, R. J. W., Van Eck, N. J., Van Leeuwen, T. N., Van Raan, A. F. J., Visser, M. S., \& Wouters, P. (2012a). The Leiden Ranking 2011/2012: Data collection, indicators, and interpretation. Journal of the American Society for Information Science and Technology, 63, 2419-2432.

Waltman, L., \& Schreiber, M. (2013). On the calculation of percentile-based bibliometric indicators. Journal of the American Society for Information Science and Technology, 64, 372-379.

Zitt M., Ramana-Rahari, S., and Bassecoulard, E. (2005). Relativity of Citation Performance and Excellence Measures: From Cross-field to Cross-scale Effects of Field-Normalization. Scientometrics, 63, 373-401. 


\section{APPENDIX}

\section{The G8 system}

As indicated in the text, let $x_{j}$ be the set of top $X \%$ most cited articles in cluster $j$ in the G8 system, and let $X^{G 8}=\cup_{j}\left\{x_{j}\right\}$ be the union of all such sets, so that the number of articles in $X^{G 8}, N^{X}$, is the $X \%$ of $N .^{5}$ Let $X_{v}^{G 8}$ be the set of articles in $X^{G 8}$ assigned to $v$ categories in the $\operatorname{WoS}$ system, where $v=1, \ldots, 6$, and let $N_{v}$ be the number of articles in $X_{v}^{G 8}$, so that $X^{G 8}=\cup_{\nu}$ $\left\{X_{v}^{G 8}\right\}$ and $N^{X}=\Sigma_{v} N_{v}$. For $v \geq 2$, we need to expand the set $X_{v}^{G 8}$ as follows. For any $v \geq 2$, denote by $X_{v}^{G 8}(\mathbf{E})$ the $v$-replica of $X_{v}^{G 8}$, so that $\left|X_{v}^{G 8}(\mathbf{E})\right|=v N_{v}$. For example, if $v=2$ and $X_{v}^{G 8}=\left(c_{v 1}, \ldots, c_{v i}, \ldots, c_{v N v}\right)$, we have

$$
X_{2}^{G 8}(\mathbf{E})=\left(c_{v i}, c_{v i}, \ldots, c_{v i}, c_{v i}, \ldots, c_{v N_{v}}, c_{v N_{v}}\right)
$$

with $\left|X_{2}^{G 8}(\mathbf{E})\right|=2 N_{2}$.

\section{The WoS system}

As indicated in the text, let $e_{k}$ be the citation distribution of category $k$ in the WoS system, $k=1, \ldots, 236$, and let $E=\cup_{k}$ $\left\{\boldsymbol{e}_{k}\right\}$ be the overall citation distribution. Every distinct article in $C$ can be assigned to $v$ categories in the WoS system with $v$ $\in\{1, \ldots, 6\}$. Therefore, every element of citation distribution $e_{k}$ consists of a number of citations, say $e_{k b}$, and a weight $w_{k b}=1 / v$ for some $v \in\{1, \ldots, 6\}$. Let $n_{k}=\Sigma_{b} w_{k b}$ be the number of articles in so that $N=\Sigma_{k} n_{k}=\Sigma_{k} \Sigma_{b} w_{k b}$.

Let $y_{k}$ be the set of top $X \%$ most cited articles in category $k$ in the WoS system, and let $X^{\mathbb{W o S}}=\cup_{k}\left\{y_{k}\right\}$ be the union of all such sets, so that the number of articles in $X^{W o S}$ is $N^{X}$, namely, the $X \%$ of $N$. For any $v \in\{1, \ldots, 6\}$, let $X_{v}^{W o s}$ be the set of elements in $X^{W o s}$ with weight equal to $1 / v$, so that $X^{W o s}=\cup_{v}\left\{X_{v}^{W o s}\right\}$. Let $M_{v}$ be the number of elements in $X_{v}^{W o s}$, so that we can represent this set by $X_{v}^{W o S}=\left\{(1 / v) e_{v l} l=1, \ldots, M_{v}\right\}$, and $N^{X}=\Sigma_{v}\left(M_{v} / v\right)$. Finally, for any $v$ we need the citation distribution consisting of the citations received by the elements in $X_{v}^{W o S}$, that is, for any $v$, we need

$$
X_{v}^{W o S}(\mathbf{D})=\left\{e_{v b} l=1, \ldots, M_{v}\right\} .
$$

\section{The intersections}

Let $\quad \bigcap_{1}=X_{1}^{G 8} \cap X_{1}^{W o s}(\mathbf{D})$,

and for any $v \geq 2$, define the intersections

$$
\bigcap_{v}=X_{v}^{G 8}(\mathbf{E}) \cap X_{v}^{W o S}(\mathbf{D}) .
$$

Let $I_{v}$ be the number of articles in $\bigcap_{v}, v=1, \ldots, 6$. The number of articles common to the sets $X^{G 8}$ and $X^{W o S}$ is $I=\Sigma_{v}\left(I_{v} / v\right)$. Therefore, the difference between the G8 and the WoS systems according to the $X$ criterion can be expressed as $100\left(N^{X}\right.$ I) $/ N^{X}$.

\section{AN EXAMPLE}

Assume there are 12 distinct articles and four clusters in system G8:

$c_{1}=(0,1,3,12), N_{1}=4 ; \quad c_{2}=(1,6), N_{2}=2 ; \quad c_{3}=(4,8), N_{3}=2 ; \quad c_{4}=(0,5,7,9), N_{4}=4$.

Assume that there are three categories, and that the distinct articles with citations 3, 5, and 7 are assigned to the three of them. Let $n_{k}$ be the number of articles in category $k, k=1,2,3$. The three categories are:

${ }_{5}$ To simplify matters, we shall assume that all $x_{j}$ consists of a whole number of distinct articles. This assumption will be dropped in the example. 


$$
\begin{aligned}
& \boldsymbol{e}_{1}=(1,1 / 3 \text { of } 3,1 / 3 \text { of } 5,1 / 3 \text { of } 7), n_{1}=2, \\
& \boldsymbol{e}_{2}=(0,0,1 / 3 \text { of } 3,4,1 / 3 \text { of } 5,6,1 / 3 \text { of } 7,8), n_{2}=6, \\
& \boldsymbol{e}_{3}=(1,1 / 3,1 / 3 \text { of } 5,1 / 3 \text { of } 7,9,12), n_{3}=4 . \\
& \text { If } X=50 \%, \text { then } \\
& x_{1}=(3,12), \quad x_{2}=(6), \quad x_{3}=(8), \quad x_{4}=(7,9),
\end{aligned}
$$

so that $X^{G 8}=(3,6,7,8,9,12)$, and $N^{X}=6$. Note that $X^{G 8}=X_{1}^{G 8} \cup X_{3}^{G 8}$, where $X_{1}^{G 8}=(6,8,9,12)$, and $X_{3}^{G 8}=(3,7)$. Therefore, $X_{3}{ }^{G 8}(\mathbf{E})=(3,3,3,7,7,7)$.

Similarly,

$$
y_{1}=(1 / 3 \text { of } 3,1 / 3 \text { of } 5,1 / 3 \text { of } 7), \quad y_{2}=(1 / 3 \text { of } 4,1 / 3 \text { of } 5,6,1 / 3 \text { of } 7,8), \quad y_{3}=(9,12) \text {, }
$$

so that $\quad X^{W o S}=(1 / 3$ of $3,1 / 3$ of $4,1 / 3$ of $5,1 / 3$ of $5,6,1 / 3$ of $7,1 / 3$ of $7,8,9,12)$.

Note that $X_{1}{ }^{W o S}(\mathbf{D})=(1 / 3$ of $4,6,8,9,12)$, and $X_{3}{ }^{W o S}(\mathbf{D})=(3,5,5,7,7)$.

Observe that:

$$
\begin{aligned}
& \cap_{1}=X_{1}^{G 8} \cap X_{1}{ }^{W o S}(\mathbf{D})=(6,8,9,12) \\
& \text { and } \cap_{3}=X_{3}^{G 8}(\mathbf{E}) \cap X_{3}{ }^{W o s}(\mathbf{D})=(3,7,7) \text {, } \\
& \text { so that } \quad I=I_{1}+I_{3} / 3=4+1=5 .
\end{aligned}
$$

Therefore, the difference between the G8 and the WoS systems when $X=50 \%$ is $100(12-5) / 12=58.3 \%$. 
Table A. University rankings according to the Top 1\% indicator in the WoS and G8 classification systems, ordered by the G8 indicator values

Rank G8

\section{University}

1 Massachusetts Institute of Technology

2 Princeton University

3 Harvard University

4 Stanford University

5 University of California, Berkeley

6 California Institute of Technology

7 London School of Hygiene \& Tropical Medicine

8 University of California, Santa Barbara

9 University of St Andrews

10 University of Chicago

11 Yale University

12 University of California, San Francisco

13 University of Oxford

14 Northwestern University

15 University of Washington - Seattle

16 Columbia University

17 University of California, San Diego

18 New York University

19 University of Cambridge

20 University of California, Santa Cruz

21 Duke University

22 University of California, Los Angeles

23 École Polytechnique Fédérale de Lausanne

24 ETH Zurich

25 University College London

26 Carnegie Mellon University

27 Oregon Health \& Science University

28 University of Dundee

29 Rice University

30 University of Pennsylvania

31 University of Colorado Boulder

32 Georgia Institute of Technology

33 Cornell University

34 Johns Hopkins University

35 Imperial College London

36 Delft University of Technology

37 Tufts University

38 University of North Carolina at Chapel Hill

39 ParisTech - École Polytechnique

40 Emory University

41 University of Michigan

42 University of Bristol

43 University of California, Irvine

44 Icahn School of Medicine at Mount Sinai

45 Washington University in St. Louis
Value G8

(1)

3.36

3.20

2.97

2.90

2.87

2.75

2.54

2.43

2.28

2.28

2.25

2.17

2.16

2.15

2.13

2.12

2.09

2.09

2.08

2.08

2.06

2.05

2.03

2.01

2.01

1.99

1.98

1.98

1.97

1.97

1.93

1.92

1.90

1.89

1.88

1.87

1.84

1.84

1.84

1.82

1.77

1.77

1.77

1.75
Value WoS

(2)

3.77

3.71

2.88

3.14

2.53

2.81

1.58

2.52

1.70

1.89

2.15

1.83

1.75

2.19

2.27

1.99

1.93

2.00

1.93

2.03

1.96

2.09

2.11

1.74

1.62

2.44

1.56

2.17

3.47

2.05

1.89

2.39

1.92

1.86

1.75

1.34

1.92

1.96

1.44

1.66

2.04

1.59

1.91

1.68

1.66
Rank WoS

(3)

1

1
2

5

4

7

6

59

8
Re-rankings = Rank WoS-Rank G8

(4)

(2) - (1)

(5)

$-0.10$

0.35

$-0.32$

0.17

$-0.37$

$-0.06$

$-1.18$

$-0.01$

$-0.73$

$-0.39$

$-0.13$

$-0.42$

$-0.42$

0.03

0.12

$-0.14$

$-0.19$

$-0.09$

$-0.16$

$-0.05$

$-0.12$

0.04

0.05

$-0.29$

$-0.38$

0.44

$-0.43$

0.19

1.49

0.08

$-0.07$

0.46

0.00

$-0.04$

$-0.14$

$-0.54$

0.05

0.12

$-0.41$

$-0.17$

0.22

$-0.18$

0.14

$-0.09$

$-0.09$ 
46 King's College London

47 University of Texas at Austin

48 University Texas Southwestern Med Center Dallas

49 University of Nice Sophia Antipolis

50 University of Illinois at Urbana-Champaign

51 University of Minnesota, Twin Cities

52 University of Wisconsin - Madison

53 Weizmann Institute of Science

54 Trinity College, Dublin

55 University of Rochester

56 University of Utah

57 Boston University

58 University of East Anglia

59 University of Twente

60 University of Notre Dame

61 Arizona State University

62 University of California, Riverside

63 University of Pittsburgh

64 Oregon State University

65 Brown University

66 Drexel University

67 University of Edinburgh

68 University of Geneva

69 Lancaster University

70 University of Maryland, College Park

71 Pennsylvania State University

72 University of Toronto

73 University of Southampton

74 Eindhoven University of Technology

75 McMaster University

76 University of Melbourne

77 Durham University

78 University of Stuttgart

79 University of Southern California

80 University of Colorado Denver

81 Baylor College of Medicine

82 University of Virginia

83 University of Lausanne

84 Stony Brook University, State University of NY

85 Erasmus University Rotterdam

86 Ohio State University

87 University of Miami

88 Dartmouth College

89 University of Zurich

90 Case Western Reserve University

91 RWTH Aachen University

92 Technical University of Denmark

93 University of Arizona
(1)

(2)

1.75

1.73

1.73

1.72

1.70

1.69

1.68

1.67

1.66

1.66

1.66

1.65

1.64

1.63

1.61

1.60

1.57

1.57

1.55

1.55

1.54

1.53

1.52

1.52

1.51

1.50

1.48

1.48

1.48

1.47

1.47

1.47

1.45

1.45

1.45

1.45

1.44

1.44

1.43

1.41

1.41

1.40

1.40

1.40

1.38

1.38

1.38

1.37
(3)

$$
1.58
$$

1.72

1.86

1.03

1.39

1.68

1.35

1.81

2.12

1.52

1.61

1.89

1.70

1.57

2.17

1.55

2.15

1.50

1.21

1.30

1.33

1.13

1.19

1.40

1.52

1.61

1.22

1.16

1.31

1.17

1.34

1.46

1.37

1.36

1.10

1.29

1.21

1.48

1.65

1.35

1.28

1.25

1.56

1.33

1.16

1.30

1.40

1.34
(4)
58

43

36

175

79

46

84

38

17

66

54

34

45

62

13

65

16

68

118

99

92

141

125

78

67

53

116

130

95

128

89

71

80

82

149

101

121

70

50

85

102

107

64

90

134

98

76

87
(2) - (1)

(5)

$12-0.17$

$\begin{array}{ll}-4 & -0.01\end{array}$

$\begin{array}{ll}-12 & 0.13\end{array}$

$126-0.69$

$29-0.31$

$\begin{array}{ll}-5 & -0.01\end{array}$

$32 \quad-0.34$

$-15 \quad 0.13$

$\begin{array}{ll}-37 & 0.46\end{array}$

$11-0.14$

$\begin{array}{ll}-2 & -0.06\end{array}$

$\begin{array}{ll}-23 & 0.24\end{array}$

$-13 \quad 0.05$

$3 \quad-0.06$

$-47 \quad 0.56$

$4 \quad-0.05$

$\begin{array}{ll}-46 & 0.57\end{array}$

$5 \quad-0.07$

$54-0.34$

$34-0.25$

$26-0.21$

$\begin{array}{ll}74 & -0.39\end{array}$

$57 \quad-0.32$

$\begin{array}{ll}9 & -0.12\end{array}$

$\begin{array}{ll}-3 & 0.01\end{array}$

$\begin{array}{ll}-18 & 0.11\end{array}$

$44 \quad-0.26$

$57 \quad-0.32$

$\begin{array}{ll}21 & -0.17\end{array}$

$53-0.30$

$13-0.13$

$\begin{array}{ll}-6 & 0.00\end{array}$

$2 \quad-0.09$

$3-0.08$

$69-0.34$

$20-0.16$

$39-0.23$

$\begin{array}{ll}-13 & 0.04\end{array}$

$\begin{array}{ll}-34 & 0.22\end{array}$

$0 \quad-0.06$

$16-0.13$

$20-0.15$

$\begin{array}{ll}-24 & 0.15\end{array}$

$1 \quad-0.07$

$44 \quad-0.22$

$\begin{array}{ll}7 & -0.08\end{array}$

$\begin{array}{ll}-16 & 0.02\end{array}$

$\begin{array}{ll}-6 & -0.03\end{array}$ 
$\begin{array}{cccc}\text { Value G8 } & \text { Value WoS } & \text { Rank WoS } & \begin{array}{c}\text { Re-rankings = } \\ \text { Rank WoS-Rank G8 }\end{array} \\ \text { (1) } & \text { (2) } & \text { (3) } & \text { (4) }\end{array}$

(1) (2)

1.37

94 University of Exeter

(3)

1.16

135

1.36

1.06

167

96 Newcastle University

97 University of British Columbia

1.36

1.14

1.35

98 Vrije Universiteit Brussel

99 Tulane University

100 Indiana University Bloomington

1.35

1.34

1.34

1.34

1.33

1.32

1.32

1.32

1.30

1.30

1.30

1.30

1.29

1.29

1.29

1.27

1.27

1.26

1.26

1.26

1.25

1.25

1.25

1.24

1.24

1.24

1.24

1.23

1.23

1.23

1.22

1.22

1.22

1.21

1.21

1.21

1.21

1.20

1.20

1.19

1.18

1.18

1.18

1.18
1.21

0.80

1.63

1.17

1.30

1.10

0.93

1.57

1.35

1.11

1.80

1.23

1.08

1.08

1.25

1.25

1.16

1.33

1.20

1.14

1.01

1.27

1.23

1.43

1.29

1.01

1.05

1.23

1.00

1.03

1.57

1.26

1.06

1.48

1.33

1.34

0.89

1.60

1.31

1.21

1.07

0.99

1.09

0.88

0.69
Re-ranki
Rank WoS-
(4)

(2) - (1)

(5)

$41 \quad-0.21$

$\begin{array}{ll}72 & -0.31\end{array}$

$43-0.23$

$22 \quad-0.14$

$175-0.54$

$\begin{array}{ll}-48 & 0.29\end{array}$

$\begin{array}{ll}26 & -0.17\end{array}$

$\begin{array}{ll}-4 & -0.03\end{array}$

$48 \quad-0.23$

$113-0.39$

$\begin{array}{ll}-44 & 0.25\end{array}$

$\begin{array}{ll}-22 & 0.04\end{array}$

$39 \quad-0.19$

$\begin{array}{ll}-68 & 0.50\end{array}$

$\begin{array}{ll}7 & -0.07\end{array}$

$49 \quad-0.22$

$47 \quad-0.21$

$\begin{array}{ll}-3 & -0.04\end{array}$

$\begin{array}{ll}-7 & -0.03\end{array}$

$19 \quad-0.11$

$\begin{array}{ll}-23 & 0.06\end{array}$

$\begin{array}{ll}9 & -0.07\end{array}$

$21-0.12$

$69-0.25$

$\begin{array}{ll}-15 & 0.02\end{array}$

$\begin{array}{ll}-6 & -0.02\end{array}$

$\begin{array}{ll}-45 & 0.18\end{array}$

$\begin{array}{ll}-21 & 0.05\end{array}$

$\begin{array}{ll}62 & -0.23\end{array}$

$\begin{array}{ll}46 & -0.19\end{array}$

$\begin{array}{ll}-12 & 0.00\end{array}$

$\begin{array}{ll}63 & -0.23\end{array}$

$51-0.20$

$-66 \quad 0.34$

$\begin{array}{ll}-24 & 0.04\end{array}$

$36-0.16$

$-61 \quad 0.26$

$\begin{array}{ll}-38 & 0.12\end{array}$

$\begin{array}{ll}-46 & 0.13\end{array}$

$99 \quad-0.32$

$\begin{array}{ll}-79 & 0.39\end{array}$

$\begin{array}{ll}-39 & 0.11\end{array}$

$\begin{array}{ll}-19 & 0.02\end{array}$

$\begin{array}{ll}27 & -0.13\end{array}$

$55-0.20$

$\begin{array}{ll}15 & -0.09\end{array}$

$95-0.30$

$184-0.48$ 
142 Colorado State University

143 Technische Universität Berlin

144 Virginia Commonwealth University

145 University of Otago

146 University of Connecticut

147 University of Queensland

148 Michigan State University

149 University of Central Florida

150 Ludwig-Maximilians-Universität München

151 University of Vermont

152 University of Cincinnati

153 Ghent University

154 Washington State University

155 University College Dublin

156 Universität Regensburg

157 Stockholm University

158 University of Strathclyde

159 Paris Diderot University

160 University of Helsinki

161 Université Montpellier 2

162 University of Groningen

163 University of Sussex

164 Wake Forest University

165 University of Leeds

166 Politecnico di Torino

167 University of Reading

168 Texas A\&M University - College Station

169 Swedish University of Agricultural Sciences

170 University of Parma

171 University of Illinois at Chicago

172 University of Bergen

173 Medical University of Vienna

174 University of New South Wales

175 University of Trieste

176 University of Sydney

177 Joseph Fourier University

178 University of Manchester

179 North Carolina State University

180 University of Montreal

181 University of Iowa

182 University of New Mexico

183 University of Bremen

184 University of Barcelona

185 Aix-Marseille University

186 University of Delaware

187 Karolinska Institute

188 University of Ottawa

189 Leiden University
Value G8 Value WoS Rank WoS $\begin{gathered}\text { Re-rankings = } \\ \text { Rank WoS-Rank }\end{gathered}$

(1) (2)

(3)

(4)

(2) - (1)

1.17

1.06

166

1.17

0.94

213

1.17

1.09

152

1.16

0.68

330

1.16

0.94

209

1.16

1.14

1.44

138

1.16

1.15

0.97

72

198

144

320

1.15

1.11

0.70

1.24

111

171

1.15

1.04

0.75

299

248

1.14

0.86

162

153

1.14

1.14

1.14

1.14

1.13

1.13

1.13

1.12

1.12

1.12

1.12

1.12

1.12

1.12

1.12

1.11

1.11

1.10

1.10

1.10

1.10

1.10

1.07

1.09

241

238

210

133

180

269

156

208

324

192

201

281

194

173

242

276

253

274

247

237

161

1.10

148

214

233

1.10

1.10

94

265

261

195

168

179

146

110
(5)

$24 \quad-0.12$

$\begin{array}{ll}70 & -0.23\end{array}$

$8 \quad-0.07$

$185-0.49$

$63-0.21$

$\begin{array}{ll}-9 & -0.02\end{array}$

$\begin{array}{ll}-76 & 0.28\end{array}$

$49 \quad-0.18$

$\begin{array}{ll}-6 & -0.04\end{array}$

$169-0.45$

$18-0.11$

$145-0.39$

$93-0.29$

$6 \quad-0.07$

$-4 \quad-0.05$

$83-0.26$

$79-0.26$

$50-0.20$

$-28$

18

106

$-8$

43

158

25

33

112

24

2

70

103

79

99

71

60

$-17$

$-31$

34

52

$-88$

82

82

77
10

10

$-18$

$-8$

$-42$

$-79$

0.03

$-0.11$

$-0.31$

$-0.04$

$-0.18$

$-0.43$

$-0.13$

$-0.15$

$-0.33$

$-0.13$

$-0.08$

$-0.24$

$-0.31$

$-0.25$

$-0.30$

$-0.24$

$-0.22$

$-0.03$

0.00

$-0.17$

$-0.21$

0.23

$-0.26$

$-0.25$

$-0.11$

$-0.03$

$-0.07$

0.02

0.15 $\begin{array}{ll}-41 & 0.09\end{array}$ 
190 Maastricht University

191 National University of Singapore

192 Universitat Politècnica de València

193 Karlsruhe Institute of Technology

194 University of Georgia

195 University of Liverpool

196 University of Strasbourg

197 Wayne State University

198 University Claude Bernard Lyon 1

199 Monash University

200 Iowa State University

201 Technische Universität Darmstadt

202 Virginia Polytechnic Institute and State University

203 University of Bordeaux 1 Science and Technology

204 University of Duisburg-Essen

205 Johannes Gutenberg Univ Mainz

206 University of Maryland, Baltimore

207 Queen's University

208 University of Liège

209 University of Birmingham

210 Queen's University Belfast

211 George Mason University

212 University of South Carolina

213 Cardiff University

214 Gottfried Wilhelm Leibniz Universität Hannover

215 Indiana University - Purdue University Indianapolis

216 University of Gothenburg

217 Giessen University

218 University of Western Ontario

219 Aarhus University

220 Simon Fraser University

221 University of Milan Bicocca

222 University of Hamburg

223 Massey University

224 Heidelberg University

225 Friedrich-Alexander-Universität Erlangen-Nürnberg

226 University of Western Australia

227 Paris Descartes University

228 University of Bonn

229 University of Nottingham

230 University of Ulm

231 University of Pavia

232 Norwegian University of Science and Technology

233 Indian Institute of Technology Kharagpur

234 University Pierre and Marie Curie

235 University of Medicine and Dentistry of New Jersey

236 Université Bordeaux Segalen

237 University of Tübingen
Value G8 Value WoS Rank WoS $\begin{gathered}\text { Re-rankings = } \\ \text { Rank WoS-Rank G8 }\end{gathered}$

(1) (2)

(3)

(4)

(2) - (1)

1.08

0.95

1.08

207

1.40

77

106

1.08

1.25

1.23

1.03

0.81

0.91

0.67

0.83

0.94

1.21

0.82

1.00

1.07

0.70

0.97

1.04

1.11

0.80

0.66

0.89

0.97

1.43

0.78

0.67

1.15

0.83

0.51

0.73

0.95

0.75

0.70

0.85

0.91

0.84

1.25

0.95

0.60

0.96

1.01

0.68

0.85

0.91

0.73

0.84

1.00

0.92

0.95
(5)

$\begin{array}{ll}17 & -0.14\end{array}$

$\begin{array}{ll}-114 & 0.32\end{array}$

$\begin{array}{ll}-86 & 0.17\end{array}$

$\begin{array}{ll}-79 & 0.15\end{array}$

$-16 \quad-0.05$

$\begin{array}{ll}76 & -0.26\end{array}$

$27 \quad-0.16$

$139-0.39$

$\begin{array}{ll}66 & -0.23\end{array}$

$12-0.12$

$65-0.23$

$-15 \quad-0.05$

$\begin{array}{ll}-43 & 0.02\end{array}$

$118-0.35$

$\begin{array}{ll}-6 & -0.08\end{array}$

$\begin{array}{ll}-36 & 0.00\end{array}$

$\begin{array}{ll}-60 & 0.06\end{array}$

$69-0.25$

$129 \quad-0.38$

$21-0.15$

$\begin{array}{ll}-11 & -0.07\end{array}$

$-138 \quad 0.40$

77

123

$-79$

47

175

89

$-14$

8

100

32

$-1$

35

$-116$

$-20$

134

$-26$

$-44$

99

19

$-12$

76

26

$-46$

$-18$

$-33$
$-0.26$

$-0.36$

0.12

$-0.19$

$-0.50$

$-0.28$

$-0.06$

$-0.26$

$-0.31$

$-0.16$

$-0.10$

$-0.16$

0.24

$-0.06$

$-0.40$

$-0.04$

0.01

$-0.32$

$-0.14$

$-0.08$

$-0.26$

$-0.15$

0.01

$-0.07$

$-0.03$
$-80 \quad 0.15$ 
Value G8 Value WoS Rank WoS $\begin{gathered}\text { Re-rankings = } \\ \text { Rank WoS-Rank G8 }\end{gathered}$

(1) (2)

238 University of Perugia

0.98

0.68

(3)

(4)

332

0.98

0.87

244

240 Aalto University

241 Auburn University

242 University of South Florida at Tampa

0.98

0.97

0.97

0.97

0.96

244 University of Adelaide

245 Vienna University of Technology

246 Politecnico di Milano

247 Université Catholique de Louvain

248 Humboldt-Universität zu Berlin

249 City University of Hong Kong

250 Radboud University Nijmegen

251 Kansas State University

252 Universidad Autónoma de Madrid

253 University at Buffalo, State University NY

254 University of Kansas

255 Medical University of South Carolina

256 Temple University

257 University of Padova

258 University of Leicester

259 Ruhr-Universität Bochum

260 University of Cologne

261 Laval University

262 University of Tennessee

263 Paul Sabatier University

264 Lund University

265 Hebrew University of Jerusalem

266 Thomas Jefferson University

267 Freie Universität Berlin

268 University of Calgary

269 University of Antwerp

270 University of Alberta

271 University of Waterloo

272 Chalmers University of Technology

273 University of the Witwatersrand

274 University of Victoria

275 Saarland University

276 University of Münster

277 University of Nebraska - Lincoln

278 University of Missouri

279 Martin Luther University of Halle-Wittenberg

280 University of Florida

281 University of Freiburg

282 Medical College of Wisconsin

283 University of Guelph

284 Chinese University of Hong Kong

285 Technical University of Lisbon

\subsection{6}

0.96

0.96

0.95

0.95

0.94

0.94

0.94

0.94

0.93

0.93

0.92

0.92

0.92

0.92

0.91

0.91

0.91

0.91

0.91

0.90

0.90

0.90

0.89

0.89

0.89

0.89

0.88

0.88

0.88

0.88

0.88

0.87

0.87

0.87

0.86

0.86

0.86

0.86

0.85

0.85
0.45

0.70

1.16

0.81

0.73

0.98

0.54

0.84

0.86

1.02

1.17

1.12

0.65

0.68

1.03

0.87

0.90

0.74

0.96

0.66

0.85

1.01

1.14

0.73

0.91

0.94

0.93

0.91

0.68

0.89

0.74

0.79

0.77

0.52

0.82

0.75

0.88

0.80

1.02

0.60

0.97

1.13

0.65

0.85

0.77

0.59
405

323

131

270

311

196

385

262

249

181

127

143

346

328

176

243

229

304

203

340

258

183

140

306

224

212

217

227

331

230

303

286

293

388

267

301

236

275

182

364

197

142

345

252

294

367
(2) - (1)

(5)

$94-0.30$

$5-0.11$

$165-0.52$

$82-0.28$

$\begin{array}{ll}-111 & 0.19\end{array}$

$27 \quad-0.15$

$\begin{array}{ll}67 & -0.24\end{array}$

-49 0.01

$139-0.42$

$15-0.12$

$\begin{array}{ll}1 & -0.09\end{array}$

$\begin{array}{ll}-68 & 0.07\end{array}$

$\begin{array}{ll}-123 & 0.23\end{array}$

$\begin{array}{ll}-108 & 0.17\end{array}$

$94-0.29$

$\begin{array}{ll}75 & -0.25\end{array}$

$\begin{array}{ll}-78 & 0.10\end{array}$

$-12 \quad-0.05$

$-27 \quad-0.03$

$47 \quad-0.18$

$\begin{array}{ll}-55 & 0.04\end{array}$

$81-0.26$

$\begin{array}{ll}-2 & -0.07\end{array}$

$\begin{array}{ll}-78 & 0.10\end{array}$

$\begin{array}{ll}-122 & 0.23\end{array}$

$43-0.17$

$\begin{array}{ll}-40 & 0.01\end{array}$

$\begin{array}{ll}-53 & 0.03\end{array}$

$\begin{array}{ll}-49 & 0.02\end{array}$

$\begin{array}{ll}-40 & 0.01\end{array}$

$63-0.22$

-39 $\quad 0.00$

$33-0.15$

$15-0.10$

$21-0.11$

$115-0.36$

$\begin{array}{ll}-7 & -0.07\end{array}$

$26-0.13$

$-40 \quad 0.01$

$\begin{array}{ll}-2 & -0.07\end{array}$

$-96 \quad 0.15$

$\begin{array}{ll}85 & -0.27\end{array}$

-83 0.11

$\begin{array}{ll}-139 & 0.27\end{array}$

$63-0.21$

$\begin{array}{ll}-31 & 0.00\end{array}$

$\begin{array}{ll}10 & -0.08\end{array}$

$82-0.26$ 
286 Nanyang Technological University

287 University of Würzburg

288 University of Alabama at Birmingham

289 Universidade Nova de Lisboa

290 Henri Poincaré University

291 Hong Kong University of Science and Technology

292 Umeå University

293 Technische Universität Dresden

294 Ewha Womans University

295 University of Rennes 1

296 Pohang University of Science and Technology

297 University of Bologna

298 Heinrich Heine Univ Düsseldorf

299 Uppsala University

300 University of Wollongong

301 Dalhousie University

302 University of Oslo

303 Oklahoma State University - Stillwater

304 University of Kentucky

305 University of Cape Town

306 Hannover Medical School

307 University of Santiago de Compostela

308 University of Texas Medical Branch at Galveston

309 University of Surrey

310 Southeast University

311 University of Aveiro

312 Louisiana State University

313 Technion - Israel Institute of Technology

314 University of Tokyo

315 Montpellier 1 University

316 Macquarie University

317 University of Jena

318 University of Porto

319 Universidad Autónoma de Barcelona

320 Harbin Institute of Technology

321 Loughborough University

322 Peking University

323 University of Valencia

324 Otto-von-Guericke University Magdeburg

325 Clemson University

326 University of Louisville

327 Dalian University of Technology

328 Kiel University

329 KTH Royal Institute of Technology

330 University of Genoa

331 University of Torino

332 University of Florence

333 Lanzhou University
Value G8 Value WoS Rank WoS $\begin{gathered}\text { Re-rankings = } \\ \text { Rank WoS-Rank }\end{gathered}$

(1) (2)

(3)

(4)

(2) - (1)

0.85

1.08

159

0.85

0.79

283

0.85

0.88

239

0.84

0.91

225

0.84

0.59

370

0.84

1.09

0.70

155

319

0.83

0.91

226

234

347

0.83

0.83

0.82

0.81

0.80

0.80

0.80

0.80

0.80

0.80

0.80

0.80

0.79

0.79

0.79

0.78

0.78

0.78

0.78

0.77

0.77

0.77

0.77

0.77

0.76

0.76

0.75

0.75

0.75

0.74

0.74

0.74

0.74

0.74

0.73

0.73

0.72

0.72

0.72

0.89

0.64

1.20

123

296

172

308

163

362

292

353

246

268

228

174

356

360

57

316

295

343

312

190

278

339

280

289

377

402

221

357

401

272

298

219

291

318

341

368

282

285
(5)

$\begin{array}{ll}-127 & 0.23\end{array}$

$\begin{array}{ll}-4 & -0.06\end{array}$

$\begin{array}{ll}-49 & 0.03\end{array}$

$\begin{array}{ll}-64 & 0.07\end{array}$

$80 \quad-0.26$

$\begin{array}{ll}-136 & 0.24\end{array}$

$27-0.13$

$\begin{array}{ll}-67 & 0.08\end{array}$

$\begin{array}{ll}52 & -0.19\end{array}$

$\begin{array}{ll}-173 & 0.38\end{array}$

$-1 \quad-0.06$

$\begin{array}{ll}-126 & 0.23\end{array}$

$9 \quad-0.07$

$\begin{array}{ll}-137 & 0.26\end{array}$

$61-0.21$

$-10 \quad-0.03$

$50 \quad-0.19$

$\begin{array}{ll}-58 & 0.07\end{array}$

$\begin{array}{ll}-37 & 0.02\end{array}$

$\begin{array}{ll}-78 & 0.10\end{array}$

$\begin{array}{ll}-133 & 0.24\end{array}$

$48 \quad-0.19$

$51-0.19$

$-253 \quad 0.81$

$\begin{array}{ll}5 & -0.07\end{array}$

$-17 \quad-0.01$

$30 \quad-0.12$

$\begin{array}{ll}-2 & -0.05\end{array}$

$\begin{array}{ll}-125 & 0.23\end{array}$

$\begin{array}{ll}-38 & 0.02\end{array}$

$22-0.11$

$\begin{array}{ll}-38 & 0.03\end{array}$

$\begin{array}{ll}-30 & 0.02\end{array}$

$57 \quad-0.21$

$81 \quad-0.29$

$-101 \quad 0.16$

$34-0.14$

$\begin{array}{ll}77 & -0.27\end{array}$

$\begin{array}{ll}-53 & 0.07\end{array}$

$\begin{array}{ll}-28 & 0.01\end{array}$

$-108 \quad 0.18$

$\begin{array}{ll}-37 & 0.03\end{array}$

$-11-0.03$

$\begin{array}{ll}11 & -0.07\end{array}$

$\begin{array}{ll}37 & -0.13\end{array}$

$\begin{array}{ll}-50 & 0.07\end{array}$

$\begin{array}{ll}-48 & 0.07\end{array}$
$-60 \quad 0.05$ 
334 University Paris-Est Créteil Val de Marne

335 University of Rostock

336 Hong Kong Polytechnic University

337 University of Lisbon

338 University of Milan

339 Amirkabir University of Technology

340 West Virginia University

341 Tel Aviv University

342 University of Turku

343 Central South University

344 University of Tasmania

345 Universität Leipzig

346 University of Manitoba

347 University of Modena and Reggio Emilia

348 Complutense University

349 University of Warsaw

350 Innsbruck Medical University

351 Aristotle University of Thessaloniki

352 University of Eastern Finland

353 Tsinghua University

354 University College Cork

355 Stellenbosch University

356 University of Oklahoma

357 Tokyo Institute of Technology

358 Queensland University of Technology

359 National Technical University of Athens

360 Tokyo Medical and Dental University

361 Osaka University

362 Kyoto University

363 University of Mississippi

364 Sharif University of Technology

365 University of Naples Federico II

366 Ben-Gurion University of the Negev

367 Banaras Hindu University

368 Xiamen University

369 University of Zaragoza

370 Federal University of Rio de Janeiro

371 University of Burgundy

372 Pontifical Catholic University of Chile

373 Indian Institute of Science

374 National Sun Yat-sen University

375 Bar-Ilan University

376 University of Science Malaysia

377 Texas Tech University

378 East China University of Science and Technology

379 University of KwaZulu-Natal

380 Università Cattolica del Sacro Cuore

381 University of Pisa
Value G8 Value WoS Rank WoS $\begin{array}{r}\text { Re-rankings = } \\ \text { Rank WoS-Rank }\end{array}$

(1) (2)

(3)

(4)

(2) - (1)

0.72

1.09

151

0.72

0.72

313

0.71

0.68

327

0.71

0.71

0.30

452

314

240

0.71

0.71

0.70

0.69

0.69

0.69

0.69

0.69

0.68

0.68

0.67

0.67

0.67

0.66

0.65

0.65

0.65

0.64

0.64

0.64

0.64

0.64

0.63

0.63

0.63

0.62

0.61

0.61

0.61

0.60

0.60

0.60

0.60

0.60

0.60

0.60

0.59

0.59

0.59

0.58

0.58

0.58

0.57

0.88

0.53

0.73

387

310

350

355

413

284

374

372

363

389

245

435

352

215

255

441

376

288

442

451

344

335

305

408

412

398

434

410

256

365

453

342

333

386

395

476

81

315

302

251

417

366
(5)

$-183$

$-22$

$-9$

115

$-24$

$-99$

47

$-31$

8

12

69

$-61$

28

25

15

40

$-105$

84

0

$-138$

$-99$

86

20

$-69$

84

92

$-16$

$-26$

$-57$

45

48

33

68

43

$-112$

$-4$

83

$-29$

$-39$

13

21

101

$-295$

$-62$

$-76$

$-128$

37

$-15$
0.38

0.00

$-0.03$

$-0.41$

0.01

0.17

$-0.18$

0.03

$-0.07$

$-0.09$

$-0.26$

0.10

$-0.13$

$-0.10$

$-0.08$

$-0.16$

0.20

$-0.31$

$-0.04$

0.28

0.20

$-0.31$

$-0.09$

0.14

$-0.31$

$-0.33$

0.01

0.04

0.10

$-0.18$

$-0.19$

$-0.13$

$-0.25$

$-0.17$

0.25

$-0.01$

$-0.30$

0.06

0.08

$-0.06$

$-0.10$

$-0.38$

0.77

0.13

0.16

0.28

$-0.17$

0.02 
382 University of Seville

383 Nanjing University

384 Shandong University

385 Lomonosov Moscow State University

386 Korea Advanced Institute of Science and Technology

387 York University

388 University of Oviedo

389 Sun Yat-sen University

390 Tohoku University

391 Nagoya University

392 University of Bari Aldo Moro

393 University of Oulu

394 Universidad de Granada

395 Linköping University

396 University of Patras

397 University of the Basque Country

398 University of Coimbra

399 Kyushu University

400 Keio University

401 Sapienza University of Rome

402 University of Tehran

403 East China Normal University

404 National and Kapodistrian University of Athens

405 National Taiwan University

406 Chonbuk National University

407 University of Nantes

408 University of Rome Tor Vergata

409 Shanghai Jiao Tong University

410 University of Saskatchewan

411 Griffith University

412 National Tsing Hua University

413 University of Palermo

414 University of Ljubljana

415 Pusan National University

416 Nankai University

417 Yonsei University

418 Seoul National University

419 Chonnam National University

420 Istanbul University

421 Kanazawa University

422 Flinders University

423 Fudan University

424 University of Lübeck

425 Kobe University

426 Federal University of Santa Catarina

427 Universidade Federal de Minas Gerais

428 University of Tsukuba

429 State University of Campinas
Value G8 Value WoS Rank WoS $\begin{gathered}\text { Re-rankings = } \\ \text { Rank WoS-Rank G8 }\end{gathered}$

(1) (2)

(3)

(4)

(2) - (1)

0.57

0.54

383

0.56

0.68

334

0.56

0.48

400

0.36

432

0.55

0.62

351

354

416

0.54

0.53

0.53

0.53

0.52

0.51

0.51

0.51

0.50

0.50

0.50

0.50

0.50

0.50

0.49

0.48

0.48

0.48

0.47

0.47

0.47

0.47

0.47

0.46

0.46

0.46

0.46

0.45

0.45

0.45

0.45

0.44

0.43

0.43

0.43

0.43

0.42

0.42

0.42

0.41

0.41

0.41

0.41

1.21

0.55

0.46

0.40

0.57

0.51

0.64

0.33

0.60

0.36

0.40

0.49

0.40

0.17

0.45

0.32

0.54

0.63

0.50

0.50

0.60

0.55

0.59

0.54

0.46

0.31

0.28

1.17

0.51

0.71

0.41

0.25

0.34

0.51

0.76

0.48

0.39

0.10

0.34

0.42

0.28
(5)

$-0.03$

$\begin{array}{ll}-49 & 0.11\end{array}$

$16-0.08$

$47 \quad-0.20$

$\begin{array}{ll}-35 & 0.07\end{array}$

$\begin{array}{ll}-33 & 0.06\end{array}$

$28 \quad-0.13$

$\begin{array}{ll}-267 & 0.67\end{array}$

$\begin{array}{ll}-12 & 0.02\end{array}$

$12-0.07$

$29-0.12$

$\begin{array}{ll}-20 & 0.05\end{array}$

$\begin{array}{ll}-4 & 0.01\end{array}$

$\begin{array}{ll}-47 & 0.14\end{array}$

$47-0.17$

$\begin{array}{ll}-39 & 0.10\end{array}$

$35-0.14$

$21-0.10$

$\begin{array}{ll}-3 & -0.01\end{array}$

$\begin{array}{ll}18 & -0.09\end{array}$

$87-0.32$

$3-0.03$

$42-0.16$

$\begin{array}{ll}-21 & 0.06\end{array}$

$\begin{array}{ll}-57 & 0.16\end{array}$

$\begin{array}{ll}-11 & 0.02\end{array}$

$\begin{array}{ll}-14 & 0.03\end{array}$

$-50 \quad 0.13$

$\begin{array}{ll}-30 & 0.08\end{array}$

$\begin{array}{ll}-40 & 0.12\end{array}$

$\begin{array}{ll}-31 & 0.08\end{array}$

$\begin{array}{ll}-9 & 0.00\end{array}$

$36-0.15$

$\begin{array}{ll}-287 & 0.72\end{array}$

-24 0.06

$-101 \quad 0.26$

$\begin{array}{ll}-1 & -0.03\end{array}$

$47 \quad-0.18$

$\begin{array}{ll}17 & -0.09\end{array}$

$\begin{array}{ll}-31 & 0.08\end{array}$

$-126 \quad 0.33$

$\begin{array}{ll}-25 & 0.06\end{array}$

$\begin{array}{ll}-2 & -0.03\end{array}$

$\begin{array}{ll}70 & -0.31\end{array}$

$9 \quad-0.07$

$\begin{array}{ll}-13 & 0.01\end{array}$

$31-0.12$
$43-0.17$ 
Value G8 Value WoS Rank WoS

(1)

430 Waseda University

431 University of Murcia

432 Indian Institute of Technology Madras

433 Hanyang University

434 University of Belgrade

435 University of Siena

436 University of Zagreb

437 University of Science and Technology of China

438 Hokkaido University

439 Technical University of Madrid

440 National Cheng Kung University

441 Hacettepe University

442 Cairo University

443 Federal University of Rio Grande do Sul

444 University of Pretoria

445 National Chung Hsing University

446 Hiroshima University

447 Saint Petersburg State University

448 Jagiellonian University in Krakow

449 Zhejiang University

450 Korea University

451 University of Buenos Aires

452 Kyungpook National University

453 University of Catania

454 National Chiao Tung University

455 Kyung Hee University

456 Wuhan University

457 Mahidol University

458 Tongji University

459 Chulalongkorn University

460 Okayama University

461 Ankara University

462 Universidade de São Paulo

463 Sungkyunkwan University

464 National Central University

465 University of Science and Technology Beijing

466 National Autonomous University of Mexico

467 Chiba University

468 Charles University in Prague

469 Xi'an Jiaotong University

470 South China University of Technology

471 Huazhong University of Science and Technology

472 Tianjin University

473 Inha University

474 Jilin University

475 Federal University of Paraná

476 University of Ulsan

477 Universidade Estadual Paulista
(2)

(3)

0.40

0.40

0.39

0.38

0.38

0.37

0.37

0.37

0.37

0.36

0.36

0.35

0.35

0.35

0.35

0.34

0.34

0.34

0.34

0.34

0.34

0.34

0.34

0.33

0.33

0.33

0.33

0.33

0.32

0.32

0.32

0.30

0.30

0.29

0.29

0.29

0.29

0.28

0.28

0.28

0.27

0.26

0.25

0.25

0.24

0.24

0.24

0.24
0.30

0.33

0.28

0.28

0.18

0.38

0.28

0.99

0.27

0.19

0.56

0.19

0.13

0.32

0.23

0.85

0.30

0.18

0.38

0.39

0.59

0.22

0.38

0.22

0.55

0.25

0.42

0.32

0.22

0.54

0.19

0.19

0.29

0.32

0.29

0.44

0.25

0.38

0.18

0.34

0.79

0.20

0.45

0.12

0.69

0.20

0.38

0.17
Re-rankings $=$ Rank WoS-Rank G8

(4)

454

440

459

462

484

424

461

191

464

479

375

483

493

445

471

257

455

486

429

422

369

472

425

475

379

468

414

448

474

382

481

480

457

447

456

411

469

430

485

439

279

478

407

495

326

477

426

487
(2) - (1)

(5)

$24 \quad-0.10$

$9 \quad-0.06$

$27-0.11$

$29-0.10$

$50-0.20$

$\begin{array}{ll}-11 & 0.01\end{array}$

$25-0.09$

$\begin{array}{ll}-246 & 0.62\end{array}$

$26-0.10$

$\begin{array}{ll}40 & -0.17\end{array}$

$\begin{array}{ll}-65 & 0.20\end{array}$

$\begin{array}{ll}42 & -0.17\end{array}$

$51-0.22$

$2-0.02$

$27 \quad-0.11$

$\begin{array}{ll}-188 & 0.50\end{array}$

$\begin{array}{ll}9 & -0.05\end{array}$

$39-0.17$

$\begin{array}{ll}-19 & 0.04\end{array}$

$\begin{array}{ll}-27 & 0.05\end{array}$

$\begin{array}{ll}-81 & 0.25\end{array}$

$21-0.11$

$\begin{array}{ll}-27 & 0.05\end{array}$

$22-0.11$

$\begin{array}{ll}-75 & 0.22\end{array}$

$13-0.08$

$\begin{array}{ll}-42 & 0.09\end{array}$

$\begin{array}{ll}-9 & -0.01\end{array}$

$16-0.10$

$\begin{array}{ll}-77 & 0.22\end{array}$

$21-0.13$

$19 \quad-0.10$

$\begin{array}{ll}-5 & -0.01\end{array}$

$\begin{array}{ll}-16 & 0.03\end{array}$

$\begin{array}{ll}-8 & 0.00\end{array}$

$\begin{array}{ll}-54 & 0.15\end{array}$

$3-0.04$

$\begin{array}{ll}-37 & 0.09\end{array}$

$17 \quad-0.10$

$\begin{array}{ll}-30 \quad 0.06 \\ -101 & 0.52\end{array}$

$-191 \quad 0.52$

$\begin{array}{ll}7 & -0.07\end{array}$

$\begin{array}{ll}-65 & 0.20\end{array}$

$22-0.13$

$\begin{array}{ll}-148 & 0.44\end{array}$

$2 \quad-0.04$

$\begin{array}{ll}-50 & 0.14\end{array}$

$10-0.07$ 
478 China Agricultural University

479 Tehran University of Medical Sciences

480 Middle East Technical University

481 Chang Gung University

482 University of Chile

483 Chungnam National University

484 Federal University of São Paulo

485 National University of La Plata

486 Ege University

487 Beijing Normal University

488 Sichuan University

489 National Yang-Ming University

490 Konkuk University

491 Federal University of Viçosa

492 University of Malaya

493 King Saud University

494 Nihon University

495 Gazi University

496 Shanghai University

497 Northwestern Polytechnical University

498 Hunan University

499 Tarbiat Modares University

500 Catholic University of Korea

Value G8 Value WoS Rank WoS $\begin{array}{r}\text { Re-rankings = } \\ \text { Rank WoS-Rank }\end{array}$

(1)

Average

$\mathrm{SD}$

CV
0.23

0.23

0.23

0.22

0.22

0.22

0.22

0.22

0.21

0.20

0.20

0.19

0.19

0.18

0.17

0.16

0.16

0.16

0.16

0.15

0.13

0.11

0.08

(2)

0.34

0.07

0.38

0.17

0.17

0.26

0.14

0.24

0.31

0.33

0.38

0.28

0.38

0.14

0.22

0.09

0.10

0.13

0.44

0.01

0.78

0.19

0.27

0.98

0.55

0.56
(3) 437

499

428

488

490

466

492

470

449

444

431

463

427

491

473

498

497

494

409

500

287

482

465
(4)

$-41$

20

$-52$

7

8

$-17$

8

$-15$

$-37$

$-43$

$-57$

$-26$

$-63$

0

$-19$

5

3

$-1$

$-87$

3

$-211$

$-17$

$-35$
(5)

(2) - (1)

0.11

$-0.16$

0.15

$-0.05$

$-0.05$

0.05

$-0.08$

0.02

0.10

0.13

0.18

0.08

0.20

$-0.04$

0.05

$-0.08$

$-0.06$

$-0.03$

0.28

$-0.14$

0.66

0.08

0.20 
Table B. University rankings according to the Top 1\% indicator in the G6 and G8 classification systems, ordered by the G8 indicator values

\section{Rank G8}

University

1 Massachusetts Institute of Technology

2 Princeton University

3 Harvard University

4 Stanford University

5 University of California, Berkeley

6 California Institute of Technology

7 London School of Hygiene \& Tropical Medicine

8 University of California, Santa Barbara

9 University of St Andrews

10 University of Chicago

11 Yale University

12 University of California, San Francisco

13 University of Oxford

14 Northwestern University

15 University of Washington - Seattle

16 Columbia University

17 University of California, San Diego

18 New York University

19 University of Cambridge

20 University of California, Santa Cruz

21 Duke University

22 University of California, Los Angeles

23 École Polytechnique Fédérale de Lausanne

24 ETH Zurich

25 University College London

26 Carnegie Mellon University

27 Oregon Health \& Science University

28 University of Dundee

29 Rice University

30 University of Pennsylvania

31 University of Colorado Boulder

32 Georgia Institute of Technology

33 Cornell University

34 Johns Hopkins University

35 Imperial College London

36 Delft University of Technology

37 Tufts University

38 University of North Carolina at Chapel Hill

39 ParisTech - École Polytechnique

40 Emory University

41 University of Michigan

42 University of Bristol

43 University of California, Irvine

44 Icahn School of Medicine at Mount Sinai

45 Washington University in St. Louis

\section{Value G8}

(1)

3.86

3.36

3.20

2.97

2.90

2.87

2.75

2.54

2.43

2.28

2.28

2.25

2.17

2.16

2.15

2.13

2.12

2.09

2.09

2.08

2.08

2.06

2.05

2.03

2.01

2.01

1.99

1.98

1.98

1.97

1.97

1.93

1.92

1.90

1.89

1.88

1.87

1.84

1.84

1.84

1.82

1.77

1.77

1.77

1.75
Value G6

(2)

3.77

3.71

2.88

3.14

2.53

2.81

1.58

2.52

1.70

1.89

2.15

1.83

1.75

2.19

2.27

1.99

1.93

2.00

1.93

2.03

1.96

2.09

2.11

1.74

1.62

2.44

1.56

2.17

3.47

2.05

1.89

2.39

1.92

1.86

1.75

1.34

1.92

1.96

1.44

1.66

2.04

1.59

1.91

1.68

1.66
Rank G6

(3)
Re-rankings =
Rank G6-Rank G8

(4)
(2) - (1)

(5)

$-0.10$

0.35

$-0.32$

0.17

$-0.37$

$-0.06$

$-1.18$

$-0.01$

$-0.73$

$-0.39$

$-0.13$

$-0.42$

$-0.42$

0.03

0.12

$-0.14$

$-0.19$

$-0.09$

$-0.16$

$-0.05$

$-0.12$

0.04

0.05

$-0.29$

$-0.38$

0.44

$-0.43$

0.19

1.49

0.08

$-0.07$

0.46

0.00

$-0.04$

$-0.14$

$-0.54$

0.05

0.12

$-0.41$

$-0.17$

0.22

$-0.18$

0.14

$-0.09$

$-0.09$ 
Rank G8

University

46 King's College London

47 University of Texas at Austin

48 University Texas Southwestern Med Center Dallas

49 University of Nice Sophia Antipolis

50 University of Illinois at Urbana-Champaign

51 University of Minnesota, Twin Cities

52 University of Wisconsin - Madison

53 Weizmann Institute of Science

54 Trinity College, Dublin

55 University of Rochester

56 University of Utah

57 Boston University

58 University of East Anglia

59 University of Twente

60 University of Notre Dame

61 Arizona State University

62 University of California, Riverside

63 University of Pittsburgh

64 Oregon State University

65 Brown University

66 Drexel University

67 University of Edinburgh

68 University of Geneva

69 Lancaster University

70 University of Maryland, College Park

71 Pennsylvania State University

72 University of Toronto

73 University of Southampton

74 Eindhoven University of Technology

75 McMaster University

76 University of Melbourne

77 Durham University

78 University of Stuttgart

79 University of Southern California

80 University of Colorado Denver

81 Baylor College of Medicine

82 University of Virginia

83 University of Lausanne

84 Stony Brook University, State University of NY

85 Erasmus University Rotterdam

86 Ohio State University

87 University of Miami

88 Dartmouth College

89 University of Zurich

90 Case Western Reserve University

91 RWTH Aachen University

92 Technical University of Denmark

93 University of Arizona
Value G8 Value G6 Rank G6

(1)

(2)

1.75

1.73

1.73

1.72

1.70

1.69

1.68

1.67

1.66

1.66

1.66

1.65

1.64

1.63

1.61

1.60

1.57

1.57

1.55

1.55

1.54

1.53

1.52

1.52

1.51

1.50

1.48

1.48

1.48

1.47

1.47

1.47

1.45

1.45

1.45

1.45

1.44

1.44

1.43

1.41

1.41

1.40

1.40

1.40

1.38

1.38

1.38

1.37
(3)

1.58

1.72

1.86

1.03

1.39

1.68

1.35

1.81

2.12

1.52

1.61

1.89

1.70

1.57

2.17

1.55

2.15

1.50

1.21

1.30

1.33

1.13

1.19

1.40

1.52

1.61

1.22

1.16

1.31

1.17

1.34

1.46

1.37

1.36

1.10

1.29

1.21

1.48

1.65

1.35

1.28

1.25

1.56

1.33

1.16

1.30

1.40

1.34
Re-rankings = Rank G6-Rank G8

(4)
(2) - (1)

(5)

$12-0.17$

$-4 \quad-0.01$

$\begin{array}{ll}-12 & 0.13\end{array}$

$126-0.69$

$29 \quad-0.31$

$\begin{array}{ll}-5 & -0.01\end{array}$

$32 \quad-0.34$

$\begin{array}{ll}-15 & 0.13\end{array}$

$\begin{array}{ll}-37 & 0.46\end{array}$

$11 \quad-0.14$

$-2 \quad-0.06$

$\begin{array}{ll}-23 & 0.24\end{array}$

$\begin{array}{ll}-13 & 0.05\end{array}$

$3 \quad-0.06$

$\begin{array}{ll}-47 & 0.56\end{array}$

$4 \quad-0.05$

$\begin{array}{ll}-46 & 0.57\end{array}$

$5 \quad-0.07$

$54 \quad-0.34$

$34-0.25$

$26-0.21$

$\begin{array}{ll}74 & -0.39\end{array}$

$57 \quad-0.32$

$9 \quad-0.12$

$\begin{array}{ll}-3 & 0.01\end{array}$

$-18 \quad 0.11$

$44 \quad-0.26$

$57 \quad-0.32$

$21 \quad-0.17$

$53-0.30$

$13-0.13$

$\begin{array}{ll}-6 & 0.00\end{array}$

$2 \quad-0.09$

$3-0.08$

$69 \quad-0.34$

$20 \quad-0.16$

$39 \quad-0.23$

$-13 \quad 0.04$

$\begin{array}{ll}-34 & 0.22\end{array}$

$0 \quad-0.06$

$16-0.13$

$20 \quad-0.15$

$-24 \quad 0.15$

$1 \quad-0.07$

$44 \quad-0.22$

$\begin{array}{ll}7 & -0.08\end{array}$

$-16 \quad 0.02$
-6

$\begin{array}{ll}-6 & -0.03\end{array}$ 
94 University of Exeter

95 Katholieke Universiteit Leuven

96 Newcastle University

97 University of British Columbia

98 Vrije Universiteit Brussel

99 Tulane University

100 Indiana University Bloomington

101 University of California, Davis

102 Florida State University

103 University of Auckland

104 University of Massachusetts Amherst

105 Vanderbilt University

106 Australian National University

107 University of Massachusetts Medical School

108 George Washington University

109 University of Glasgow

110 Technische Universität München

111 Utrecht University

112 University of Amsterdam

113 Wageningen University and Research Centre

114 Northeastern University

115 University of Sheffield

116 McGill University

117 Georgetown University

118 VU University Amsterdam

119 University of Bath

120 University of Aberdeen

121 Purdue University - Lafayette

122 Philipps-Universität Marburg

123 Université Libre de Bruxelles

124 University of Copenhagen

125 University of Ferrara

126 University of Hawaii, Manoa

127 Rutgers State University at New Brunswick

128 University of York

129 Queen Mary, University of London

130 Yeshiva University

131 University of Paris-Sud 11

132 Goethe University Frankfurt

133 University of Göttingen

134 University Texas Health Sci. Ctr.

135 University of Hong Kong

136 University of Basel

137 University of Vienna

138 University of Southern Denmark

139 University of Bern

140 University of Warwick

141 Universitat Politècnica de Catalunya

\section{Value G8}

(1)

1.37

1.36

1.36

1.35

1.35

1.34

1.34

1.34

1.33

1.32

1.32

1.32

1.30

1.30

1.30

1.30

1.29

1.29

1.29

1.27

1.27

1.26

1.26

1.26

1.25

1.25

1.25

1.24

1.24

1.24

1.24

1.23

1.23

1.23

1.22

1.22

1.22

1.21

1.21

1.21

1.21

1.20

1.20

1.19

1.18

1.18

1.18

1.18
Value G6

(2)

1.16

1.06

1.14

1.21

0.80

1.63

1.17

1.30

1.10

0.93

1.57

1.35

1.11

1.80

1.23

1.08

1.08

1.25

1.25

1.16

1.33

1.20

1.14

1.01

1.27

1.23

1.43

1.29

1.01

1.05

1.23

1.00

1.03

1.57

1.26

1.06

1.48

1.33

1.34

0.89

1.60

1.31

1.21

1.07

0.99

1.09

0.88

0.69
Rank G6

(3)

135

167

139

119

273

51

126

97

150

216

60

83

145

39

115

158

157

108

105

132

91

124

137

186

103

113

75

100

184

169

112

188

177

61

104

165

69

93

86

232

55

96

117

164

193

154

235

325
Re-rankings = Rank G6-Rank G8

(4)

(2) - (1)

(5)

$41 \quad-0.21$

$72 \quad-0.31$

$43-0.23$

$22 \quad-0.14$

$175-0.54$

$\begin{array}{ll}-48 & 0.29\end{array}$

$\begin{array}{ll}26 & -0.17\end{array}$

$-4 \quad-0.03$

$48 \quad-0.23$

$113-0.39$

$-44 \quad 0.25$

$\begin{array}{ll}-22 & 0.04\end{array}$

$39 \quad-0.19$

$\begin{array}{ll}-68 & 0.50\end{array}$

$7 \quad-0.07$

$49 \quad-0.22$

$47 \quad-0.21$

$\begin{array}{ll}-3 & -0.04\end{array}$

$\begin{array}{ll}-7 & -0.03\end{array}$

$19 \quad-0.11$

$\begin{array}{ll}-23 & 0.06\end{array}$

$\begin{array}{ll}9 & -0.07\end{array}$

$21 \quad-0.12$

$69 \quad-0.25$

$\begin{array}{ll}-15 & 0.02\end{array}$

$\begin{array}{ll}-6 & -0.02\end{array}$

$-45 \quad 0.18$

$\begin{array}{ll}-21 & 0.05\end{array}$

$62-0.23$

$46 \quad-0.19$

$\begin{array}{ll}-12 & 0.00\end{array}$

$63-0.23$

$51-0.20$

$\begin{array}{ll}-66 & 0.34\end{array}$

$\begin{array}{ll}-24 & 0.04\end{array}$

$36 \quad-0.16$

$\begin{array}{ll}-61 & 0.26\end{array}$

$\begin{array}{ll}-38 & 0.12\end{array}$

$\begin{array}{ll}-46 & 0.13\end{array}$

$99 \quad-0.32$

$\begin{array}{ll}-79 & 0.39\end{array}$

-39 $\quad 0.11$

$\begin{array}{ll}-19 & 0.02\end{array}$

$27 \quad-0.13$

$55 \quad-0.20$

$15-0.09$

$95-0.30$

$184-0.48$ 
Rank G8

University

142 Colorado State University

143 Technische Universität Berlin

144 Virginia Commonwealth University

145 University of Otago

146 University of Connecticut

147 University of Queensland

148 Michigan State University

149 University of Central Florida

150 Ludwig-Maximilians-Universität München

151 University of Vermont

152 University of Cincinnati

153 Ghent University

154 Washington State University

155 University College Dublin

156 Universität Regensburg

157 Stockholm University

158 University of Strathclyde

159 Paris Diderot University

160 University of Helsinki

161 Université Montpellier 2

162 University of Groningen

163 University of Sussex

164 Wake Forest University

165 University of Leeds

166 Politecnico di Torino

167 University of Reading

168 Texas A\&M University - College Station

169 Swedish University of Agricultural Sciences

170 University of Parma

171 University of Illinois at Chicago

172 University of Bergen

173 Medical University of Vienna

174 University of New South Wales

175 University of Trieste

176 University of Sydney

177 Joseph Fourier University

178 University of Manchester

179 North Carolina State University

180 University of Montreal

181 University of Iowa

182 University of New Mexico

183 University of Bremen

184 University of Barcelona

185 Aix-Marseille University

186 University of Delaware

187 Karolinska Institute

188 University of Ottawa

189 Leiden University

\section{Value G8}

(1)

1.17

1.17

1.17

1.16

1.16

1.16

1.16

1.15

1.15

1.15

1.15

1.15

1.15

1.14

1.14

1.14

1.14

1.14

1.14

1.13

1.13

1.13

1.12

1.12

1.12

1.12

1.12

1.12

1.12

1.12

1.11

1.11

1.10

1.10

1.10

1.10

1.10

1.10

1.10

1.10

1.10

1.09

1.09

1.09

1.09

1.09

1.09

1.09
Value G6

(2)

1.06

0.94

1.09

0.68

0.94

1.14

1.44

0.97

1.11

0.70

1.24

1.04

0.75

0.86

1.07

1.09

0.88

0.88

0.94

1.16

1.02

0.81

1.08

0.95

0.70

0.99

0.96

0.79

0.99

1.03

0.87

0.80

0.85

0.80

0.86

0.88

1.07

1.10

0.93

0.89

1.33

0.83

0.84

0.98

1.05

1.02

1.11

1.24
Rank G6

(3)

166

213

152

330

209

138

72

198

144

320

111

171

299

248

162

153

241

238

210

133

180

269

156

208

324

192

201

281

194

173

242

276

253

274

247

237

161

148

214

233

94

265

261

195

168

179

146

110
Re-rankings = Rank G6-Rank G8

(4)

(2) - (1)

(5)

$24 \quad-0.12$

$\begin{array}{ll}70 & -0.23\end{array}$

$\begin{array}{ll}8 & -0.07\end{array}$

$185-0.49$

$63-0.21$

$\begin{array}{ll}-9 & -0.02\end{array}$

$\begin{array}{ll}-76 & 0.28\end{array}$

$49-0.18$

$-6 \quad-0.04$

$169-0.45$

$-41 \quad 0.09$
-18

$18 \quad-0.11$

$145-0.39$

$93 \quad-0.29$

$\begin{array}{ll}6 & -0.07\end{array}$

$-4 \quad-0.05$

$83 \quad-0.26$

$79 \quad-0.26$

$50 \quad-0.20$

$\begin{array}{ll}-28 & 0.03\end{array}$

$18 \quad-0.11$

$106-0.31$

$\begin{array}{ll}-8 & -0.04\end{array}$

$43 \quad-0.18$

$158 \quad-0.43$

$25-0.13$

$33-0.15$

$112-0.33$

$24 \quad-0.13$

$2-0.08$

$70 \quad-0.24$

$103-0.31$

$79 \quad-0.25$

$99 \quad-0.30$

$71 \quad-0.24$

$60 \quad-0.22$

$-17 \quad-0.03$

$\begin{array}{ll}-31 & 0.00\end{array}$

$34 \quad-0.17$

$52 \quad-0.21$

$\begin{array}{ll}-88 & 0.23\end{array}$

$82 \quad-0.26$

$\begin{array}{ll}77 & -0.25\end{array}$

$10 \quad-0.11$

$-18 \quad-0.03$

$\begin{array}{ll}-8 & -0.07\end{array}$

$\begin{array}{ll}-42 & 0.02\end{array}$

$\begin{array}{ll}-79 & 0.15\end{array}$ 
Rank G8

University

190 Maastricht University

191 National University of Singapore

192 Universitat Politècnica de València

193 Karlsruhe Institute of Technology

194 University of Georgia

195 University of Liverpool

196 University of Strasbourg

197 Wayne State University

198 University Claude Bernard Lyon 1

199 Monash University

200 Iowa State University

201 Technische Universität Darmstadt

202 Virginia Polytechnic Institute and State Univ.

203 University of Bordeaux 1 Sci. \& Technol.

204 University of Duisburg-Essen

205 Johannes Gutenberg Univ Mainz

206 University of Maryland, Baltimore

207 Queen's University

208 University of Liège

209 University of Birmingham

210 Queen's University Belfast

211 George Mason University

212 University of South Carolina

213 Cardiff University

214 Gottfried Wilhelm Leibniz Universität

215 Indiana University - Purdue University

216 University of Gothenburg

217 Giessen University

218 University of Western Ontario

219 Aarhus University

220 Simon Fraser University

221 University of Milan Bicocca

222 University of Hamburg

223 Massey University

224 Heidelberg University

225 Friedrich-Alexander-Univ. Erlangen-Nürnberg

226 University of Western Australia

227 Paris Descartes University

228 University of Bonn

229 University of Nottingham

230 University of Ulm

231 University of Pavia

232 Norwegian University of Sci. \& Technol.

233 Indian Institute of Technology Kharagpur

234 University Pierre and Marie Curie

235 University of Medicine and Dentistry of New Jersey

236 Université Bordeaux Segalen

237 University of Tübingen

\author{
(1)
}

Value G8

1.08

1.08

1.08

1.08

1.07

1.07

1.07

1.06

1.06

1.06

1.06

1.06

1.05

1.05

1.05

1.05

1.05

1.04

1.04

1.04

1.04

1.04

1.04

1.04

1.03

1.03

1.02

1.02

1.02

1.01

1.01

1.01

1.01

1.01

1.01

1.01

1.01

1.00

1.00

1.00

0.99

0.99

0.99

0.99

0.99

0.99

0.98

0.98

\section{Value G6}

(2)

0.95

1.40

1.25

1.23

1.03

0.81

0.91

0.67

0.83

0.94

1.21

0.82

1.00

1.07

0.70

0.97

1.04

1.11

0.80

0.66

0.89

0.97

1.43

0.78

0.67

1.15

0.83

0.51

0.73

0.95

0.75

0.70

0.85

0.91

0.84

1.25

0.95

0.60

0.96

1.01

0.68

0.85

0.91

0.73

0.84

1.00

0.92

0.95
Rank G6

(3)

207

77

106

114

178

271

223

336

264

211

120

266

187

160

322

199

170

147

277

338

231

200

74

290

337

136

263

392

307

205

300

321

254

222

259

109

206

361

202

185

329

250

220

309

260

189

218

204
Re-rankings = Rank G6-Rank G8

(4)

(2) - (1)

(5)

$17 \quad-0.14$

$\begin{array}{ll}-114 & 0.32\end{array}$

$\begin{array}{ll}-86 & 0.17\end{array}$

$\begin{array}{ll}-79 & 0.15\end{array}$

$\begin{array}{ll}-16 & -0.05\end{array}$

$\begin{array}{ll}76 & -0.26\end{array}$

$27-0.16$

$139 \quad-0.39$

$66-0.23$

$12 \quad-0.12$

$\begin{array}{ll}-80 & 0.15\end{array}$

$\begin{array}{ll}65 & -0.23\end{array}$

$-15 \quad-0.05$

$\begin{array}{ll}-43 & 0.02\end{array}$

$118-0.35$

$\begin{array}{ll}-6 & -0.08\end{array}$

$\begin{array}{ll}-36 & 0.00\end{array}$

$-60 \quad 0.06$

$69-0.25$

$129 \quad-0.38$

$21 \quad-0.15$

$\begin{array}{ll}-11 & -0.07\end{array}$

$-138 \quad 0.40$

$\begin{array}{ll}77 & -0.26\end{array}$

$123-0.36$

$\begin{array}{ll}-79 & 0.12\end{array}$

$47 \quad-0.19$

$175-0.50$

$89-0.28$

$\begin{array}{ll}-14 & -0.06\end{array}$

$80 \quad-0.26$

$100 \quad-0.31$

$32 \quad-0.16$

$-1 \quad-0.10$

$35 \quad-0.16$

$-116 \quad 0.24$

$-20 \quad-0.06$

$134 \quad-0.40$

$-26-0.04$

$\begin{array}{ll}-44 & 0.01\end{array}$

$99 \quad-0.32$

$19-0.14$

$-12-0.08$

$\begin{array}{ll}76 & -0.26\end{array}$

$26--0.15$

$\begin{array}{ll}-46 & 0.01\end{array}$

$\begin{array}{ll}-18 & -0.07\end{array}$

$\begin{array}{ll}-33 & -0.03\end{array}$ 
Rank G8

University

238 University of Perugia

239 University of Houston

240 Aalto University

241 Auburn University

242 University of South Florida at Tampa

243 University of Newcastle

244 University of Adelaide

245 Vienna University of Technology

246 Politecnico di Milano

247 Université Catholique de Louvain

248 Humboldt-Universität zu Berlin

249 City University of Hong Kong

250 Radboud University Nijmegen

251 Kansas State University

252 Universidad Autónoma de Madrid

253 University at Buffalo, State University NY

254 University of Kansas

255 Medical University of South Carolina

256 Temple University

257 University of Padova

258 University of Leicester

259 Ruhr-Universität Bochum

260 University of Cologne

261 Laval University

262 University of Tennessee

263 Paul Sabatier University

264 Lund University

265 Hebrew University of Jerusalem

266 Thomas Jefferson University

267 Freie Universität Berlin

268 University of Calgary

269 University of Antwerp

270 University of Alberta

271 University of Waterloo

272 Chalmers University of Technology

273 University of the Witwatersrand

274 University of Victoria

275 Saarland University

276 University of Münster

277 University of Nebraska - Lincoln

278 University of Missouri

279 Martin Luther University of Halle-Wittenberg

280 University of Florida

281 University of Freiburg

282 Medical College of Wisconsin

283 University of Guelph

284 Chinese University of Hong Kong

285 Technical University of Lisbon

\section{Value G8}

(1)

0.98

0.98

0.98

0.97

0.97

0.97

0.96

0.96

0.96

0.96

0.95

0.95

0.94

0.94

0.94

0.94

0.93

0.93

0.92

0.92

0.92

0.92

0.91

0.91

0.91

0.91

0.91

0.90

0.90

0.90

0.89

0.89

0.89

0.89

0.88

0.88

0.88

0.88

0.88

0.87

0.87

0.87

0.86

0.86

0.86

0.86

0.85

0.85

\section{Value G6}

(2)

0.68

0.87

0.45

0.70

1.16

0.81

0.73

0.98

0.54

0.84

0.86

1.02

1.17

1.12

0.65

0.68

1.03

0.87

0.90

0.74

0.96

0.66

0.85

1.01

1.14

0.73

0.91

0.94

0.93

0.91

0.68

0.89

0.74

0.79

0.77

0.52

0.82

0.75

0.88

0.80

1.02

0.60

0.97

1.13

0.65

0.85

0.77

0.59
Rank G6

(3) 332

244

405

323

131

270

311

196

385

262

249

181

127

143

346

328

176

243

229

304

203

340

258

183

140

306

224

212

217

227

331

230

303

286

293

388

267

301

236

275

182

364

197

142

345

252

294

367
Re-rankings = Rank G6-Rank G8

(4)

(2) - (1)

(5)

$94-0.30$

$5-0.11$

$165-0.52$

$82-0.28$

$\begin{array}{ll}-111 & 0.19\end{array}$

$27 \quad-0.15$

$\begin{array}{ll}67 & -0.24\end{array}$

$\begin{array}{ll}-49 & 0.01\end{array}$

$139-0.42$

$15-0.12$

$1 \quad-0.09$

$\begin{array}{ll}-68 & 0.07\end{array}$

$\begin{array}{ll}-123 & 0.23\end{array}$

$\begin{array}{ll}-108 & 0.17\end{array}$

$94 \quad-0.29$

$75 \quad-0.25$

$\begin{array}{ll}-78 & 0.10\end{array}$

$\begin{array}{ll}-12 & -0.05\end{array}$

$\begin{array}{ll}-27 & -0.03\end{array}$

$47 \quad-0.18$

$\begin{array}{ll}-55 & 0.04\end{array}$

$81-0.26$

$\begin{array}{ll}-2 & -0.07\end{array}$

$-78=0.10$

$\begin{array}{ll}-122 & 0.23\end{array}$

$43 \quad-0.17$

$\begin{array}{ll}-40 & 0.01\end{array}$

$\begin{array}{ll}-53 & 0.03\end{array}$

$\begin{array}{ll}-49 & 0.02\end{array}$

$\begin{array}{ll}-40 & 0.01\end{array}$

$\begin{array}{ll}63 & -0.22\end{array}$

$\begin{array}{ll}-39 & 0.00\end{array}$

$33-0.15$

$15-0.10$

$21 \quad-0.11$

$115 \quad-0.36$

$\begin{array}{ll}-7 & -0.07\end{array}$

$26-0.13$

$\begin{array}{ll}-40 & 0.01\end{array}$

$\begin{array}{ll}-2 & -0.07\end{array}$

$\begin{array}{ll}-96 & 0.15\end{array}$

$85 \quad-0.27$

$\begin{array}{ll}-83 & 0.11\end{array}$

$\begin{array}{ll}-139 & 0.27\end{array}$

$63-0.21$

$\begin{array}{ll}-31 & 0.00\end{array}$

$10 \quad-0.08$

$82 \quad-0.26$ 
286 Nanyang Technological University

287 University of Würzburg

288 University of Alabama at Birmingham

289 Universidade Nova de Lisboa

290 Henri Poincaré University

291 Hong Kong University of Sci. \& Technol.

292 Umeå University

293 Technische Universität Dresden

294 Ewha Womans University

295 University of Rennes 1

296 Pohang University of Sci. \& Technol.

297 University of Bologna

298 Heinrich Heine Univ Düsseldorf

299 Uppsala University

300 University of Wollongong

301 Dalhousie University

302 University of Oslo

303 Oklahoma State University - Stillwater

304 University of Kentucky

305 University of Cape Town

306 Hannover Medical School

307 University of Santiago de Compostela

308 University of Texas Med Branch

309 University of Surrey

310 Southeast University

311 University of Aveiro

312 Louisiana State University

313 Technion - Israel Institute of Technology

314 University of Tokyo

315 Montpellier 1 University

316 Macquarie University

317 University of Jena

318 University of Porto

319 Universidad Autónoma de Barcelona

320 Harbin Institute of Technology

321 Loughborough University

322 Peking University

323 University of Valencia

324 Otto-von-Guericke University Magdeburg

325 Clemson University

326 University of Louisville

327 Dalian University of Technology

328 Kiel University

329 KTH Royal Institute of Technology

330 University of Genoa

331 University of Torino

332 University of Florence

333 Lanzhou University

\section{Value G8}

(1)

0.85

0.85

0.85

0.84

0.84

0.84

0.84

0.83

0.83

0.83

0.83

0.82

0.81

0.80

0.80

0.80

0.80

0.80

0.80

0.80

0.80

0.79

0.79

0.79

0.78

0.78

0.78

0.78

0.77

0.77

0.77

0.77

0.77

0.76

0.76

0.75

0.75

0.75

0.74

0.74

0.74

0.74

0.74

0.73

0.73

0.72

0.72

0.72

\section{Value G6}

(2)

1.08

0.79

0.88

0.91

0.59

1.09

0.70

0.91

0.89

0.64

1.20

0.76

1.04

0.73

1.07

0.60

0.77

0.61

0.87

0.81

0.90

1.03

0.60

0.60

1.59

0.71

0.77

0.65

0.72

1.00

0.79

0.66

0.79

0.78

0.55

0.46

0.91

0.60

0.48

0.81

0.75

0.92

0.77

0.70

0.66

0.59

0.79

0.79
Rank G6

(3)

159

283

239

225

370

155

319

226

234

347

123

296

172

308

163

362

292

353

246

268

228

174

356

360

57

316

295

343

312

190

278

339

280

289

377

402

221

357

401

272

298

219

291

318

341

368

282

285
Re-rankings = Rank G6-Rank G8

(4)

(5)

$\begin{array}{ll}-127 & 0.23\end{array}$

$\begin{array}{ll}-4 & -0.06\end{array}$

$\begin{array}{ll}-49 & 0.03\end{array}$

$\begin{array}{ll}-64 & 0.07\end{array}$

$80-0.26$

$\begin{array}{ll}-136 & 0.24\end{array}$

$27 \quad-0.13$

$\begin{array}{ll}-67 & 0.08\end{array}$

$\begin{array}{ll}-60 & 0.05\end{array}$

$52 \quad-0.19$

$\begin{array}{ll}-173 & 0.38\end{array}$

$-1 \quad-0.06$

$\begin{array}{ll}-126 & 0.23\end{array}$

$9 \quad-0.07$

$-137 \quad 0.26$

$\begin{array}{ll}61 & -0.21\end{array}$

$-10 \quad-0.03$

$\begin{array}{ll}50 & -0.19\end{array}$

$\begin{array}{ll}-58 & 0.07\end{array}$

$\begin{array}{ll}-37 & 0.02\end{array}$

$\begin{array}{ll}-78 & 0.10\end{array}$

$\begin{array}{ll}-133 & 0.24\end{array}$

$\begin{array}{ll}48 & -0.19\end{array}$

$51 \quad-0.19$

$-253 \quad 0.81$

$5 \quad-0.07$

$-17 \quad-0.01$

$30 \quad-0.12$

$\begin{array}{ll}-2 & -0.05\end{array}$

$\begin{array}{ll}-125 & 0.23\end{array}$

$\begin{array}{ll}-38 & 0.02\end{array}$

$22 \quad-0.11$

$\begin{array}{ll}-38 & 0.03\end{array}$

$\begin{array}{ll}-30 & 0.02\end{array}$

$57 \quad-0.21$

$81 \quad-0.29$

$-101 \quad 0.16$

$34 \quad-0.14$

$\begin{array}{ll}77 & -0.27\end{array}$

$\begin{array}{ll}-53 & 0.07\end{array}$

$-28 \quad 0.01$

$-108 \quad 0.18$

$-37 \quad 0.03$

$\begin{array}{ll}-11 & -0.03\end{array}$

$11-0.07$

$37-0.13$

$\begin{array}{ll}-50 & 0.07\end{array}$

$\begin{array}{ll}-48 & 0.07\end{array}$ 
Rank G8

University

334 University Paris-Est Créteil Val de Marne

335 University of Rostock

336 Hong Kong Polytechnic University

337 University of Lisbon

338 University of Milan

339 Amirkabir University of Technology

340 West Virginia University

341 Tel Aviv University

342 University of Turku

343 Central South University

344 University of Tasmania

345 Universität Leipzig

346 University of Manitoba

347 University of Modena and Reggio Emilia

348 Complutense University

349 University of Warsaw

350 Innsbruck Medical University

351 Aristotle University of Thessaloniki

352 University of Eastern Finland

353 Tsinghua University

354 University College Cork

355 Stellenbosch University

356 University of Oklahoma

357 Tokyo Institute of Technology

358 Queensland University of Technology

359 National Technical University of Athens

360 Tokyo Medical and Dental University

361 Osaka University

362 Kyoto University

363 University of Mississippi

364 Sharif University of Technology

365 University of Naples Federico II

366 Ben-Gurion University of the Negev

367 Banaras Hindu University

368 Xiamen University

369 University of Zaragoza

370 Federal University of Rio de Janeiro

371 University of Burgundy

372 Pontifical Catholic University of Chile

373 Indian Institute of Science

374 National Sun Yat-sen University

375 Bar-Ilan University

376 University of Science Malaysia

377 Texas Tech University

378 East China University of Sci. \& Technol.

379 University of KwaZulu-Natal

380 Università Cattolica del Sacro Cuore

381 University of Pisa
Value G8

(1)

0.72

0.72

0.71

0.71

0.71

0.71

0.71

0.70

0.69

0.69

0.69

0.69

0.69

0.68

0.68

0.67

0.67

0.67

0.66

0.65

0.65

0.65

0.64

0.64

0.64

0.64

0.64

0.63

0.63

0.63

0.62

0.61

0.61

0.61

0.60

0.60

0.60

0.60

0.60

0.60

0.60

0.59

0.59

0.59

0.58

0.58

0.58

0.57
Value G6

(2)

1.09

0.72

0.68

0.30

0.71

0.88

0.53

0.73

0.63

0.61

0.44

0.79

0.56

0.58

0.60

0.52

0.87

0.35

0.62

0.93

0.85

0.33

0.55

0.78

0.33

0.31

0.65

0.67

0.74

0.45

0.44

0.48

0.36

0.44

0.85

0.59

0.30

0.65

0.68

0.54

0.50

0.21

1.37

0.71

0.74

0.85

0.41

0.59
Rank G6

(3)

151

313

327

452

314

240

387

310

350

355

413

284

374

372

363

389

245

435

352

215

255

441

376

288

442

451

344

335

305

408

412

398

434

410

256

365

453

342

333

386

395

476

81

315

302

251

417

366
Re-rankings = Rank G6-Rank G8

(4)

(2) - (1)

(5)

$\begin{array}{ll}-183 & 0.38\end{array}$

$\begin{array}{ll}-22 & 0.00\end{array}$

$-9 \quad-0.03$

$115-0.41$

$\begin{array}{ll}-24 & 0.01\end{array}$

$\begin{array}{ll}-99 & 0.17\end{array}$

$\begin{array}{ll}47 & -0.18\end{array}$

\begin{tabular}{ll}
$-31 \quad 0.03$ \\
\hline
\end{tabular}

$8-0.07$

$12 \quad-0.09$

$69 \quad-0.26$

$\begin{array}{ll}-61 & 0.10\end{array}$

$28-0.13$

$25-0.10$

$15 \quad-0.08$

$40 \quad-0.16$

$\begin{array}{ll}-105 & 0.20\end{array}$

$84-0.31$

$0 \quad-0.04$

$\begin{array}{ll}-138 & 0.28\end{array}$

$\begin{array}{ll}-99 & 0.20\end{array}$

$86-0.31$

$20 \quad-0.09$

$\begin{array}{ll}-69 & 0.14\end{array}$

$84-0.31$

$92-0.33$

$\begin{array}{ll}-16 & 0.01\end{array}$

$\begin{array}{ll}-26 & 0.04\end{array}$

$\begin{array}{ll}-57 & 0.10\end{array}$

$45 \quad-0.18$

$\begin{array}{ll}48 & -0.19\end{array}$

$33-0.13$

$\begin{array}{ll}68 & -0.25\end{array}$

$\begin{array}{ll}43 & -0.17\end{array}$

$\begin{array}{ll}-112 & 0.25\end{array}$

$\begin{array}{ll}-4 & -0.01\end{array}$

$83 \quad-0.30$

$\begin{array}{ll}-29 & 0.06\end{array}$

$\begin{array}{ll}-39 & 0.08\end{array}$

$13-0.06$

$21-0.10$

$101-0.38$

$\begin{array}{ll}-295 & 0.77\end{array}$

$\begin{array}{ll}-62 & 0.13\end{array}$

$\begin{array}{ll}-76 & 0.16\end{array}$

$\begin{array}{ll}-128 & 0.28\end{array}$

$37 \quad-0.17$

$\begin{array}{ll}-15 & 0.02\end{array}$ 
Rank G8

University

382 University of Seville

383 Nanjing University

384 Shandong University

385 Lomonosov Moscow State University

386 Korea Advanced Institute of Sci. \& Technol.

387 York University

388 University of Oviedo

389 Sun Yat-sen University

390 Tohoku University

391 Nagoya University

392 University of Bari Aldo Moro

393 University of Oulu

394 Universidad de Granada

395 Linköping University

396 University of Patras

397 University of the Basque Country

398 University of Coimbra

399 Kyushu University

400 Keio University

401 Sapienza University of Rome

402 University of Tehran

403 East China Normal University

404 National and Kapodistrian University Athens

405 National Taiwan University

406 Chonbuk National University

407 University of Nantes

408 University of Rome Tor Vergata

409 Shanghai Jiao Tong University

410 University of Saskatchewan

411 Griffith University

412 National Tsing Hua University

413 University of Palermo

414 University of Ljubljana

415 Pusan National University

416 Nankai University

417 Yonsei University

418 Seoul National University

419 Chonnam National University

420 Istanbul University

421 Kanazawa University

422 Flinders University

423 Fudan University

424 University of Lübeck

425 Kobe University

426 Federal University of Santa Catarina

427 Universidade Federal de Minas Gerais

428 University of Tsukuba

429 State University of Campinas
Value G8 Value G6 Rank G6

(1)

0.57

(2)

0.54

(3)

0.68

0.48

0.36

0.62

0.61

0.41

1.21

0.55

0.46

0.40

0.57

0.51

0.64

0.33

0.60

0.36

0.40

0.49

0.40

0.17

0.45

0.32

0.54

0.63

0.50

0.50

0.60

0.55

0.59

0.54

0.46

0.31

0.28

1.17

0.51

0.71

0.41

0.25

0.34

0.51

0.76

0.48

0.39

0.10

0.34

0.42

0.28
Re-rankings = Rank G6-Rank G8

(4)

(2) - (1)

(5)

$$
383
$$

334

400

432

351

354

416

122

378

403

421

373

390

348

443

358

433

420

397

419

489

406

446

384

349

396

394

359

380

371

381

404

450

458

129

393

317

418

467

438

391

297

399

423

496

436

415

460
$-0.03$

$\begin{array}{ll}-49 & 0.11\end{array}$

$16-0.08$

$\begin{array}{ll}47 & -0.20\end{array}$

$\begin{array}{ll}-35 & 0.07\end{array}$

$\begin{array}{ll}-33 & 0.06\end{array}$

$28 \quad-0.13$

$\begin{array}{ll}-267 & 0.67\end{array}$

$\begin{array}{ll}-12 & 0.02\end{array}$

$\begin{array}{ll}12 & -0.07\end{array}$

$29 \quad-0.12$

$\begin{array}{ll}-20 & 0.05\end{array}$

$\begin{array}{ll}-4 & 0.01\end{array}$

$\begin{array}{ll}-47 & 0.14\end{array}$

$\begin{array}{ll}47 & -0.17\end{array}$

$\begin{array}{ll}-39 & 0.10\end{array}$

$35 \quad-0.14$

$21 \quad-0.10$

$-3 \quad-0.01$

$18 \quad-0.09$

$87 \quad-0.32$

$3-0.03$

$42-0.16$

$\begin{array}{ll}-21 & 0.06\end{array}$

$\begin{array}{ll}-57 & 0.16\end{array}$

$\begin{array}{ll}-11 & 0.02\end{array}$

$\begin{array}{ll}-14 & 0.03\end{array}$

$\begin{array}{ll}-50 & 0.13\end{array}$

$\begin{array}{ll}-30 & 0.08\end{array}$

$\begin{array}{ll}-40 & 0.12\end{array}$

$-31 \quad 0.08$

$\begin{array}{ll}-9 & 0.00\end{array}$

$36-0.15$

$\begin{array}{ll}43 & -0.17\end{array}$

$\begin{array}{ll}-287 & 0.72\end{array}$

$\begin{array}{ll}-24 & 0.06\end{array}$

$-101 \quad 0.26$

$-1 \quad-0.03$

$47 \quad-0.18$

$17 \quad-0.09$

$\begin{array}{ll}-31 & 0.08\end{array}$

$\begin{array}{ll}-126 & 0.33\end{array}$

$\begin{array}{ll}-25 & 0.06\end{array}$

$\begin{array}{ll}-2 & -0.03\end{array}$

$\begin{array}{ll}70 & -0.31\end{array}$

$\begin{array}{ll}9 & -0.07\end{array}$

$\begin{array}{ll}-13 & 0.01\end{array}$

$31-0.12$ 
Rank G8

University

430 Waseda University

431 University of Murcia

432 Indian Institute of Technology Madras

433 Hanyang University

434 University of Belgrade

435 University of Siena

436 University of Zagreb

437 University of Sci. \& Technol. of China

438 Hokkaido University

439 Technical University of Madrid

440 National Cheng Kung University

441 Hacettepe University

442 Cairo University

443 Federal University of Rio Grande do Sul

444 University of Pretoria

445 National Chung Hsing University

446 Hiroshima University

447 Saint Petersburg State University

448 Jagiellonian University in Krakow

449 Zhejiang University

450 Korea University

451 University of Buenos Aires

452 Kyungpook National University

453 University of Catania

454 National Chiao Tung University

455 Kyung Hee University

456 Wuhan University

457 Mahidol University

458 Tongji University

459 Chulalongkorn University

460 Okayama University

461 Ankara University

462 Universidade de São Paulo

463 Sungkyunkwan University

464 National Central University

465 University of Sci. \& Technol. Beijing

466 National Autonomous University of Mexico

467 Chiba University

468 Charles University in Prague

469 Xi'an Jiaotong University

470 South China University of Technology

471 Huazhong University of Sci. \& Technol.

472 Tianjin University

473 Inha University

474 Jilin University

475 Federal University of Paraná

476 University of Ulsan

477 Universidade Estadual Paulista

\section{Value G8}

(1)

0.40

0.40

0.39

0.38

0.38

0.37

0.37

0.37

0.37

0.36

0.36

0.35

0.35

0.35

0.35

0.34

0.34

0.34

0.34

0.34

0.34

0.34

0.34

0.33

0.33

0.33

0.33

0.33

0.32

0.32

0.32

0.30

0.30

0.29

0.29

0.29

0.29

0.28

0.28

0.28

0.27

0.26

0.25

0.25

0.24

0.24

0.24

0.24
Value G6

(2)

0.30

0.33

0.28

0.28

0.18

0.38

0.28

0.99

0.27

0.19

0.56

0.19

0.13

0.32

0.23

0.85

0.30

0.18

0.38

0.39

0.59

0.22

0.38

0.22

0.55

0.25

0.42

0.32

0.22

0.54

0.19

0.19

0.29

0.32

0.29

0.44

0.25

0.38

0.18

0.34

0.79

0.20

0.45

0.12

0.69

0.20

0.38

0.17
Rank G6

(3)

454

440

459

462

484

424

461

191

464

479

375

483

493

445

471

257

455

486

429

422

369

472

425

475

379

468

414

448

474

382

481

480

457

447

456

411

469

430

485

439

279

478

407

495

326

477

426

487
Re-rankings = Rank G6-Rank G8

(4)

(2) - (1)

(5)

$24-0.10$

$9 \quad-0.06$

$27-0.11$

$29-0.10$

$50 \quad-0.20$

$\begin{array}{ll}-11 & 0.01\end{array}$

$\begin{array}{ll}25 & -0.09\end{array}$

$\begin{array}{ll}-246 & 0.62\end{array}$

$26-0.10$

$40 \quad-0.17$

$\begin{array}{ll}-65 & 0.20\end{array}$

$\begin{array}{ll}42 & -0.17\end{array}$

$51-0.22$

$2 \quad-0.02$

$27 \quad-0.11$

$\begin{array}{ll}-188 & 0.50\end{array}$

$9 \quad-0.05$

$39 \quad-0.17$

$\begin{array}{ll}-19 & 0.04\end{array}$

$\begin{array}{ll}-27 & 0.05\end{array}$

$\begin{array}{ll}-81 & 0.25\end{array}$

$21 \quad-0.11$

$\begin{array}{ll}-27 & 0.05\end{array}$

$22-0.11$

$\begin{array}{ll}-75 & 0.22\end{array}$

$13-0.08$

$\begin{array}{ll}-42 & 0.09\end{array}$

$\begin{array}{ll}-9 & -0.01\end{array}$

$16 \quad-0.10$

$\begin{array}{ll}-77 & 0.22\end{array}$

$\begin{array}{ll}21 & -0.13\end{array}$

$19-0.10$

$\begin{array}{ll}-5 & -0.01\end{array}$

$\begin{array}{ll}-16 & 0.03\end{array}$

$\begin{array}{ll}-8 & 0.00\end{array}$

$\begin{array}{ll}-54 & 0.15\end{array}$

$3 \quad-0.04$

$\begin{array}{ll}-37 & 0.09\end{array}$

$17 \quad-0.10$

$\begin{array}{ll}-30 & 0.06\end{array}$

$-191 \quad 0.52$

$\begin{array}{ll}7 & -0.07\end{array}$

$\begin{array}{ll}-65 & 0.20\end{array}$

$22-0.13$

$\begin{array}{ll}-148 & 0.44\end{array}$

$2 \quad-0.04$

$\begin{array}{ll}-50 & 0.14\end{array}$

$10 \quad-0.07$ 
Rank G8

University

478 China Agricultural University

479 Tehran University of Medical Sciences

480 Middle East Technical University

481 Chang Gung University

482 University of Chile

483 Chungnam National University

484 Federal University of São Paulo

485 National University of La Plata

486 Ege University

487 Beijing Normal University

488 Sichuan University

489 National Yang-Ming University

490 Konkuk University

491 Federal University of Viçosa

492 University of Malaya

493 King Saud University

494 Nihon University

495 Gazi University

496 Shanghai University

497 Northwestern Polytechnical University

498 Hunan University

499 Tarbiat Modares University

500 Catholic University of Korea
Value G8

(1)

0.23

Average

Standard deviation

Coefficient of variation
0.23

0.23

0.22

0.22

0.22

0.22

0.22

0.21

0.20

0.20

0.19

0.19

0.18

0.17

0.16

0.16

0.16

0.16

0.15

0.13

0.11

0.08

Value G6 Rank G6

(2)

0.34

0.07

0.38

0.17

0.17

0.26

0.14

0.24

0.31

0.33

0.38

0.28

0.38

0.14

0.22

0.09

0.10

0.13

0.44

0.01

0.78

0.19

0.27

0.98

0.55

0.56
Re-rankings = Rank G6-Rank G8

(4)

(3) 437

499

428

488

490

466

492

470

449

444

431

463

427

491

473

498

497

494

409

500

287

482

465
(2) - (1)

(5)

$\begin{array}{ll}-41 & 0.11\end{array}$

$20 \quad-0.16$

$\begin{array}{ll}-52 & 0.15\end{array}$

$7-0.05$

$8-0.05$

$\begin{array}{ll}-17 & 0.05\end{array}$

$8 \quad-0.08$

$\begin{array}{ll}-15 & 0.02\end{array}$

$\begin{array}{ll}-37 & 0.10\end{array}$

$-43 \quad 0.13$

$\begin{array}{ll}-57 & 0.18\end{array}$

$\begin{array}{ll}-26 & 0.08\end{array}$

$\begin{array}{ll}-63 & 0.20\end{array}$

$0 \quad-0.04$

$-19 \quad 0.05$

$5-0.08$

$3-0.06$

$\begin{array}{ll}-1 & -0.03\end{array}$

$\begin{array}{ll}-87 & 0.28\end{array}$

$3 \quad-0.14$

$-211 \quad 0.66$

$\begin{array}{ll}-17 & 0.08\end{array}$

$\begin{array}{ll}-35 & 0.20\end{array}$ 
Table 1. Differences in \% between the top most cited articles in the G8, G6 and WoS classification systems in the dataset consisting of 3.6 million of distinct articles

\begin{tabular}{lrrr} 
& \multicolumn{3}{c}{ Most cited articles: } \\
& Top 50\% & Top 10\% & Top 1\% \\
G8 vs G6 & $9.0 \%$ & $20.4 \%$ & $31.1 \%$ \\
G8 vs WoS & $12.7 \%$ & $31.7 \%$ & $51.7 \%$ \\
G6 vs WoS & $11.2 \%$ & $27.0 \%$ & $44.7 \%$
\end{tabular}


Table 2.A. University ranking differences according to the Top $1 \%$ indicator in going from the WoS to the G8 systems

\begin{tabular}{|c|c|c|c|}
\hline & $\begin{array}{c}\text { First } 100 \\
\text { universities } \\
\text { (1) }\end{array}$ & $\begin{array}{c}\text { Remaining } 400 \\
\text { universities } \\
\text { (2) }\end{array}$ & $\begin{array}{l}\text { Total } \\
(1+2)\end{array}$ \\
\hline$>50$ positions & 11 & 153 & 164 \\
\hline $26-50$ & 21 & 111 & 132 \\
\hline $16-25$ & 15 & 52 & 67 \\
\hline $6-15$ & 22 & 54 & 76 \\
\hline $1-5$ & 25 & 28 & 53 \\
\hline Unchanged & 6 & 2 & 8 \\
\hline Total & 100 & 400 & 100 \\
\hline
\end{tabular}

Table 2.B. University differences in Top $1 \%$ values in going from the WoS to the G8 systems

\begin{tabular}{|c|c|c|c|}
\hline & $\begin{array}{c}\text { First } 100 \\
\text { universities }\end{array}$ & $\begin{array}{l}400 \text { remaining } \\
\text { universities }\end{array}$ & $\begin{array}{l}\text { WoS values } \\
\text { - G8 values }\end{array}$ \\
\hline$>0.20$ & 31 & 125 & 156 \\
\hline$>0.10$ and $\leq 0.20$ & 31 & 116 & 147 \\
\hline$>0.05$ and $\leq 0.10$ & 18 & 86 & 104 \\
\hline$\leq 0.05$ & 20 & 73 & 93 \\
\hline Total & 100 & 400 & 100 \\
\hline
\end{tabular}

Table 2.C. Main gainers and losers in the change from the WoS to the G8 system (only universities in the top 100 according to the $\mathbf{G 8}$ system are considered) according to the Top $1 \%$ indicator

\begin{tabular}{|c|c|c|c|}
\hline University & Rank G8 & Re-ranking positions & WoS $_{\text {Top 1\% }}$ - G8 Top 1\% \\
\hline \multicolumn{4}{|l|}{ Gainers } \\
\hline 1 London School of Hygiene \& Tropical Medicine & 7 & 52 & -1.18 \\
\hline 2 Delft University of Technology & 36 & 52 & -0.54 \\
\hline 3 University of Nice Sophia Antipolis & 49 & 126 & -0.69 \\
\hline 4 Oregon State University & 64 & 54 & -0.34 \\
\hline 5 University of Edinburgh & 67 & 74 & -0.39 \\
\hline 6 University of Geneva & 68 & 57 & -0.32 \\
\hline 7 University of Southampton & 73 & 57 & -0.32 \\
\hline 8 McMaster University & 75 & 53 & -0.30 \\
\hline 9 University of Colorado Denver & 80 & 69 & -0.34 \\
\hline 10 Katholieke Universiteit Leuven & 95 & 72 & -0.31 \\
\hline 11 Vrije Universiteit Brussel & 98 & 175 & -0.54 \\
\hline \multicolumn{4}{|l|}{ Losers } \\
\hline 1 Rice University & 29 & -26 & 1.49 \\
\hline
\end{tabular}


Table 3.A. University ranking differences according to the Top $10 \%$ indicator in going from the WoS to the G8 systems

\begin{tabular}{|c|c|c|c|}
\hline & $\begin{array}{c}\text { First } 100 \\
\text { universities } \\
\text { (1) }\end{array}$ & $\begin{array}{c}\text { Remaining } 400 \\
\text { universities } \\
\text { (2) }\end{array}$ & $\begin{array}{l}\text { Total } \\
(1+2)\end{array}$ \\
\hline$>50$ positions & 0 & 81 & 81 \\
\hline $26-50$ & 7 & 107 & 114 \\
\hline $16-25$ & 13 & 74 & 87 \\
\hline 6-15 & 36 & 81 & 117 \\
\hline$\leq 5$ positions & 44 & 57 & 101 \\
\hline Total & 100 & 400 & 100 \\
\hline
\end{tabular}

Table 3.B. University differences in Top 10\% values in going from the WoS to the G8 systems

\begin{tabular}{rrrr} 
& \multicolumn{1}{c}{$\begin{array}{c}\text { First 100 } \\
\text { universities }\end{array}$} & $\begin{array}{c}\text { 400 remaining } \\
\text { universities }\end{array}$ & \multicolumn{2}{c}{ WoS values } \\
$\mathbf{>}$ - G8 values
\end{tabular}

Total

100

400

100 


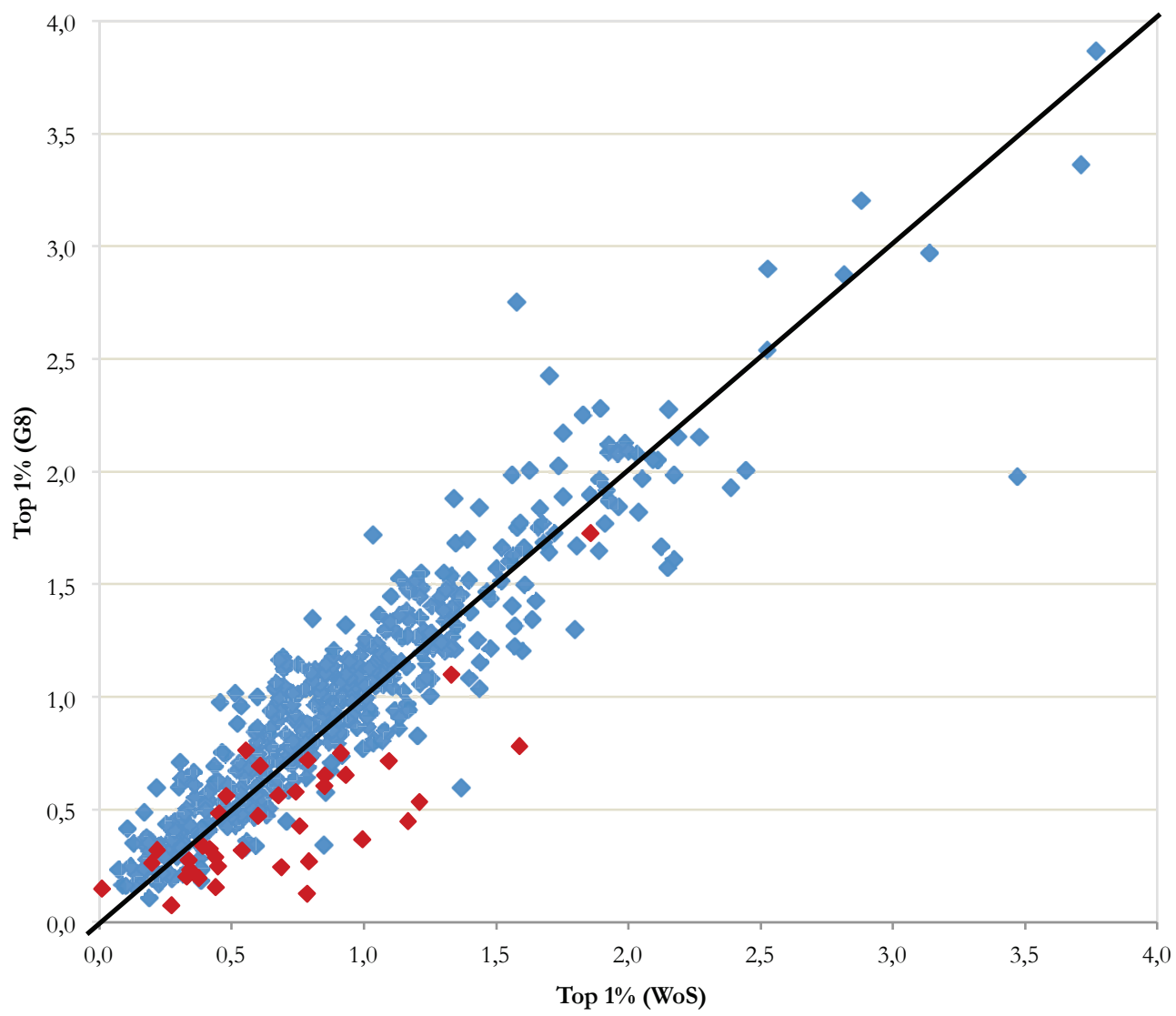

Figure 1. Scatter plot of the 500 LR universities' Top 1\% values when we use the WoS or the G8 classification systems. Chinese universities (excluding Hong Kong) are indicated in red. 
Table 4.A. University ranking differences according to the Top $1 \%$ indicator in going from the G6 to the G8 systems

\begin{tabular}{rrcrr} 
& $\begin{array}{c}\text { First 100 } \\
\text { universities }\end{array}$ & $\begin{array}{c}\text { Remaining 400 } \\
\text { universities }\end{array}$ & Total \\
\hline $\mathbf{5 0}$ positions & $(\mathbf{1})$ & $\mathbf{( 2 )}$ & $\mathbf{( 1 + 2 )}$ \\
$\mathbf{2 6 - 5 0}$ & 3 & & 91 & 3 \\
$\mathbf{1 6 - 2 5}$ & 14 & & 114 & 128 \\
$\mathbf{6 - 1 5}$ & 16 & 59 & 75 \\
$\mathbf{1 - 5}$ & 37 & 74 & 111 \\
Unchanged & 23 & 54 & 77 \\
& 7 & 8 & 15 \\
Total & & & & \\
& & & 400 & 500
\end{tabular}

Table 4.B. University differences in Top 1\% values in going from the G6 to the G8 systems

$\begin{array}{rrrr} & \begin{array}{c}\text { First 100 } \\ \text { universities }\end{array} & \begin{array}{c}\text { Remaining } \\ \text { universities }\end{array} & \text { G6 values-G8 values } \\ >\mathbf{0 . 2 0} & 29 & 52 & 81 \\ \mathbf{> 0 . 1 0} \text { and } \leq \mathbf{0 . 2 0} & 35 & 114 & 149 \\ \mathbf{> 0 . 0 5} \text { and } \leq \mathbf{0 . 1 0} & 17 & 104 & 121 \\ \mathbf{\leq 0 . 0 5} & 19 & 130 & 149 \\ & & & 500\end{array}$

Table 4.C. Main gainers and losers in the change from the G6 to the G8 system (only universities in the top 100 according to the $\mathbf{G 8}$ system are considered) according to the Top $1 \%$ indicator

\begin{tabular}{lrrr}
\multicolumn{1}{c}{ University } & Rank G8 & Re-ranking positions & G6 $_{\text {Top }}$ 1\% $^{- \text {G8 }}$ Top $_{\text {1\% }}$ \\
Gainers & & 64 & -0.51 \\
1 ParisTech - École Polytechnique & 39 & 87 & -0.31 \\
2 Technological University Denmark & 92 & 117 & -0.39 \\
3 University of Exeter & 94 & 50 & -0.16 \\
4 Vrije Universiteit Brussel & 98 & -31 & 0.59 \\
Losers & & 48 &
\end{tabular}

